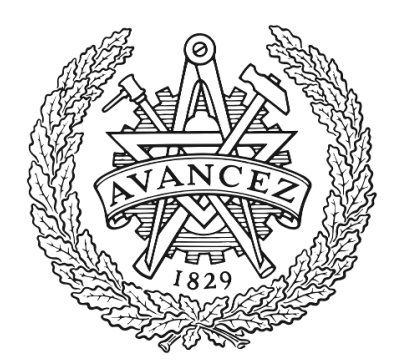

CHALMERS

\title{
Prediction of Nonlinear Distortion in Wideband Active Antenna Arrays
}

Downloaded from: https://research.chalmers.se, 2023-04-26 14:08 UTC

Citation for the original published paper (version of record):

Hausmair, K., Gustafsson, S., Sanchez Perez, C. et al (2017). Prediction of Nonlinear Distortion in Wideband Active Antenna Arrays. IEEE Transactions on Microwave Theory and Techniques, 65(11): 4550-4563. http://dx.doi.org/10.1109/TMTT.2017.2699962

N.B. When citing this work, cite the original published paper.

C2017 IEEE. Personal use of this material is permitted.

However, permission to reprint/republish this material for advertising or promotional purposes 


\title{
Prediction of Nonlinear Distortion in Wideband Active Antenna Arrays
}

\author{
Katharina Hausmair, Sebastian Gustafsson, César Sánchez-Pérez, Per N. Landin, Ulf Gustavsson, Thomas \\ Eriksson, Christian Fager
}

\begin{abstract}
In this paper we propose a technique for comprehensive analysis of nonlinear and dynamic characteristics of multiantenna transmitters (TXs). The analysis technique is enabled by the development of a Volterra series-based dual-input model for power amplifiers (PAs), which is capable of taking into account the joint effects of $P A$ nonlinearity, antenna crosstalk and mismatch for wideband modulated signals. By combining multiple instances of the PA model with linear dynamic antenna simulations we develop the analysis technique. The proposed method allows the prediction of the output signal of every antenna in an arbitrarily sized TX array, as well as the total far-field radiated wave of the $\mathrm{TX}$ for any input signal with low computational effort. A $2.12 \mathrm{GHz}$ four-element TX demonstrator based on GaAs PAs is implemented to verify simulation results with measurements. The proposed technique is a powerful tool to study hardware characteristics, as for example the effects of antenna design and element spacing. It can be used in cases where experiments are not feasible, and thus aid the development of next generation wireless systems.
\end{abstract}

Index Terms-Active antenna array, antenna crosstalk, mismatch, MIMO transmitter, power amplifier modeling

\section{INTRODUCTION}

Wireless communication systems face a steadily growing demand for higher data rates. However, the radio spectrum is a limited resource. Multiple-input multiple-output (MIMO) systems can be utilized to increase spectral efficiency [1]. For this reason, modern wireless telecommunication standards, such as LTE and Wi-Fi, include the use of multiple antennas. Largescale antenna systems, which comprise hundreds of antennas, have become a hot topic in the research community [2].

The use of several transmit paths in a transmitter (TX) increases system complexity and cost [3]. Therefore, integrated solutions, as have been used in, e.g., radar applications for many years, are preferred. Such integrated designs avoid costly components like bulky isolators between power amplifiers

This research has been carried out in GigaHertz Centre in a joint project financed by the Swedish Governmental Agency for Innovation Systems (VINNOVA), Chalmers University of Technology, Ericsson, Infineon Technologies Austria, Ampleon, National Instruments, and Saab. This project has received funding from the EMPIR programme co-financed by the Participating States and from the European Union's Horizon 2020 research and innovation programme

K. Hausmair and T. Eriksson are with the Department of Signals and Systems, Communication Systems Group, Chalmers University of Technology, 41296 Göteborg, Sweden (e-mail: \{hausmair, thomase $\} @$ chalmers.se).

C. Sánchez-Pérez was and S. Gustafsson and C. Fager are with the Department of Microtechnology and Nanoscience, Microwave Electronics Laboratory, Chalmers University of Technology, 41296 Göteborg, Sweden (e-mail: cesar.sanchez@qamcom.se, \{sebgus, christian.fager\}@chalmers.se).

P. N. Landin and U. Gustavsson are with Ericsson Research, Sweden (email: \{per.landin, ulf.gustavsson\}@ericsson.com).
(PAs) and antennas [4]. However, such designs are vulnerable to crosstalk due to mutual coupling between the antennas, and antenna mismatches. These effects, together with the nonlinear behavior of the PA, cause nonlinear distortion at the TX outputs and thus undesired radiated field properties. Predicting the output of a multi-antenna TX suffering from such distortion is essential for assessing its overall performance. It is also necessary for identifying the need for techniques to compensate for undesired effects and for the design of such techniques, like, for example, digital predistortion (DPD) [5], [6].

The authors of [7] present a low-complexity technique to model the nonlinear characteristics of the different PAs in an active antenna array system. Each PA is modeled by a combination of a core model that is common to all the PAs in the array and an individual model. The models are based on single-input single-output (SISO) wideband PA models. However, the interaction between the nonlinear behavior of the PA and the effects of antenna coupling and mismatches under wideband signal conditions cannot be described by conventional PA models used for SISO TXs. The authors of [8] take a system level oriented approach to investigate the effects of PA nonlinearity, I/Q imbalance and crosstalk in MIMO beamforming systems. They even propose a compensation method for the undesired effects. Rather than analyzing the performance of the TX hardware, the technique proposed in [8] allows to estimate the overall system performance, including the channel and receiver, in terms of average symbol error probability. However, a memoryless model is used for the PA. Hence, the analysis is not suitable for wideband signals, which require dynamic effects to be considered [9].

In [4] and [10] a hardware oriented approach is used to predict radiation patterns of active antenna arrays with direct connections between PAs and antennas. In both papers, dualinput PA models based on polyharmonic distortion (PHD) models (nonlinear scattering functions) [11], and antenna Sparameters are used to investigate the effects of mutual antenna coupling and mismatches on the behavior of PAs and on the overall performance of a TX antenna array. The proposed methods are frequency-domain based and quasi-static. Therefore, they are not suitable for analyzing multi-antenna TXs with wideband signals as used in modern wireless systems.

Recent work by Zargar et al. in [12] presents a dualinput PA model that is capable of modeling large reflections at both input and output PA ports while also taking into account dynamic effects. However, multi-antenna systems are not investigated. 
In [13], we introduced a powerful technique to predict and analyze the performance of multi-antenna TXs. It was evaluated with spectrum measurements for a two-path TX. In this work, we extend our work in [13] by presenting the derivations for the equations. We give detailed explanations of all the steps that are required to implement our technique. We have also extended our technique to account for nonconstant frequency response of the antennas, thereby extending its accuracy for wideband signal excitations. Furthermore, we now include a comprehensive experimental evaluation, where we evaluate our technique using both time- and frequencydomain measurements of a four-path TX.

With our method, it is possible to predict the output of every single antenna of an arbitrarily sized TX array for any MIMO or digital beamforming input signal scenario. Dynamic effects in multi-antenna systems can be predicted by incorporating a time-domain dual-input PA model into antenna array simulations. The PA model, which is similar to the modeling approach presented in [12], takes into account PA nonlinearity, antenna crosstalk and mismatch at the same time. While our approach is also related to the PHD-based approaches, our work includes both a dual-input PA model that is capable of considering nonlinear dynamic effects and its incorporation into multi-antenna systems. Therefore, the proposed analysis technique enables completely new possibilities to analyze hardware effects in integrated multi-antenna TXs with wideband signals. This makes it a convenient and valuable tool for the design and development of future wireless systems.

The paper is organized as follows: Section II gives an overview of multi-antenna TXs utilizing active antenna arrays and the effects that are present in such TXs. In Section III, we give the full derivation of the Volterra series-based dual-input PA model that we proposed in [13]. Then, in Section IV, we show how the proposed PA model can be used to predict the output of a TX with an arbitrary number of antenna elements. After that, we present the design of an experimental fourelement TX for measurements and simulations in Section V. Measurement results are used to validate the simulation results in Section VI. An outlook of how our work could be utilized and continued in the future is given in Section VII together with our conclusions.

\section{Multi-Antenna TX System Model}

The model is formulated in the equivalent discrete-time lowpass description, as is commonly done when modeling RF PAs [14]. The multi-antenna TX has $L$ parallel transmit paths. Each path operates in the same frequency band and consists of an RF PA which is connected to one antenna element in the transmit array. In our system model we refer only to PAs and antennas. However, if there is no in-phase/quadrature-phase (I/Q) imbalance present, this system model may represent the full multi-antenna TX chain from digital-to-analog converter to antenna. The signal $b_{2 i}(n)$ describes the PA output voltage wave of the $i$ th TX path at time step $n$. The incident wave $a_{1 i}(n)$ is the input signal to the PA of the $i$ th branch. The signal $a_{2 i}(n)$ is a wave incident to the output of the $i$ th PA.

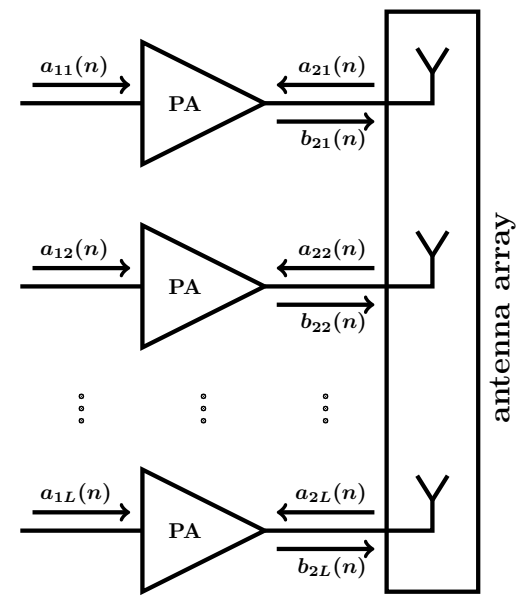

Fig. 1. Multi-antenna TX system model with $L$ transmit paths. Each path consists of one PA connected to an antenna element. All PAs are assumed to be identical and are operated in the same frequency band.

It arrives from the antenna and contains contributions from antenna crosstalk and mismatches. Finite impulse response (FIR) filters can be used to describe the relation between an incident wave $a_{2 i}(n)$ and the output signals $b_{2 i}(n)$ as

$$
\begin{aligned}
a_{2 i}(n) & =\sum_{l=1}^{L} \sum_{k=0}^{K} \lambda_{i l}(k) b_{2 l}(n-k) \\
& =\sum_{k=0}^{K}\left(\boldsymbol{\lambda}_{i}(k)\right)^{T} \mathbf{b}_{\mathbf{2}}(n-k)
\end{aligned}
$$

where $\lambda_{i l}(k)$ is the $k$ th of $K+1$ filter coefficients of the FIR filter that describes the contribution of the $l$ th antenna to the incident wave $a_{2 i}(n)$ of the $i$ th antenna, $\boldsymbol{\lambda}_{i}(k)=\left[\lambda_{i 1}(k), \ldots, \lambda_{i L}(k)\right]^{T}$ and $\mathbf{b}_{\mathbf{2}}(n-k)=\left[b_{21}(n-\right.$ $\left.k), \ldots, b_{2 L}(n-k)\right]^{T}$. The array scattering parameters (Sparameters) $\mathbf{S}_{\text {ant }}$ describe the characteristics of an antenna array in frequency domain. The time domain FIR filters in (1) can be extracted from antenna array S-parameters given over a range of frequencies. If the antennas are wideband compared to the signal bandwidth, the single-frequency S-parameters of the antenna array can be used to describe the relation between the incident waves $a_{2 i}(n)$ and the output signals $b_{2 i}(n)$ as

$$
a_{2 i}(n)=\left(\boldsymbol{\lambda}_{i}(0)\right)^{T} \mathbf{b}_{\mathbf{2}}(n)
$$

where $\boldsymbol{\lambda}_{i}(0)$ is an $L \times 1$ vector containing the $i$ th column of the $L \times L \mathrm{~S}$-parameter matrix at center frequency. This description is equivalent to the FIR filter representation for a filter with $K=0$.

In a TX without any distortion, crosstalk, or mismatch, $a_{2 i}(n)$ equals zero, while $a_{1 i}(n)$ is the signal that, amplified, would result in $b_{2 i}(n)$, radiated by the $i$ th antenna. However, realistic PAs show dynamic nonlinear behavior and in multiantenna systems also crosstalk and mismatches are present. The effects of this combined behavior result in dynamic nonlinear distortion.

In order to predict the output of a TX with multiple antennas, the joint effects of PA nonlinearity, crosstalk and mismatches have to be considered. Therefore, in the following 
section, a time-domain dynamic nonlinear PA model with two inputs, corresponding to $a_{1 i}(n)$ and $a_{2 i}(n)$, is developed. The presented model is suitable for analysis of multi-antenna TX systems with wideband input signals.

\section{PA Models FOR Multi-AntennA TX Systems}

First, the baseband description of a dual-input RF PA that considers two input signals around the same carrier frequency $f_{c}$ is derived. Similar to the model presented in [12], the resulting model is based on the Volterra series approach [15]. However, the structure of the model here is adapted to fit the description of the output signal of a PA in the presence of antenna mismatch and crosstalk. To avoid complexity issues, the presented model is then reduced following the memory polynomial approach [9]. We also show a reduced quasistatic form of the proposed model that is equivalent to PHD models [11].

\section{A. Dynamic Dual-Input PA Model}

As shown in Fig. 1, the two inputs to the $i$ th PA of a multiantenna TX are $a_{1 i}(n)$ and $a_{2 i}(n)$, while the output is denoted by $b_{2 i}(n)$. Like in a conventional single-input PA, nonlinear terms and memory effects depending on the input signal $a_{1 i}(n)$ are expected at the output of the PA. If the second input $a_{2 i}(n)$, which depends on crosstalk and mismatches, can be considered relatively small in power, only linear terms of $a_{2 i}(n)$ need to be considered [16]. Due to the dynamic behavior of the system, also past values of $a_{2 i}(n)$ may have an effect. In addition to that, the signal $a_{2 i}(n)$ mixes with the PA output in the nonlinear PA. These mixing terms also need to be considered in the model.

A Volterra series-based model that fits this structure is given in Appendix A. The resulting model for the $i$ th branch of the TX up to a nonlinear order of 3 is given by

$$
\left.\begin{array}{rl}
b_{2 i}(n)= & \sum_{m_{1}=0}^{M} \alpha_{m_{1}}^{(1)} a_{1 i}\left(n-m_{1}\right) \\
& +\sum_{m_{1}=0}^{M} \beta_{m_{1}}^{(1)} a_{2 i}\left(n-m_{1}\right) \\
& +\sum_{m_{1}=0}^{M} \sum_{m_{2}=m_{1}}^{M} \sum_{m_{3}=0}^{M} \alpha_{m_{1} m_{2} m_{3}}^{(3)} \\
& \times a_{1 i}\left(n-m_{1}\right) a_{1 i}\left(n-m_{2}\right) a_{1 i}^{*}\left(n-m_{3}\right)
\end{array}\right\}
$$

where $\alpha, \beta$, and $\gamma$ are the model coefficients, and $M$ is the memory depth. Memory effects are introduced to make the model suitable for wideband signals, where dynamic effects need to be considered [9]. The terms described by (3a) are linear dynamic effects of the PA on the input $a_{1 i}(n)$, while (3b) describes linear dynamic antenna reflection and coupling effects. In (3c), 3rd-order nonlinear dynamic effects of the PA on $a_{1 i}(n)$ are described. Finally, (3d) contains joint 3rdorder nonlinear effects which arise from mixing of mutual antenna coupling, antenna mismatches and PA nonlinearity. As explained before, only linear terms of the signal $a_{2 i}(n)$ occur with significant power. While the effects described in (3a) and (3c) are similar to the effects experienced by a PA in a SISO TX, the terms in (3b) and (3d) appear only in systems with multiple antennas. ${ }^{1}$

It can be seen that (3) contains only odd-order combinations of signals, where in each combination there is exactly one more non-conjugate term than conjugate terms. This is due to the fact that only these specific combinations will result in signal components located in the frequency band that is relevant to the description of the nonlinear system [17].

\section{B. Reduced Dual-Input PA Models}

Introducing memory according to the full Volterra-series leads to extremely high model complexity, as is demonstrated by (3), and in Appendix A. Because of the high complexity, a full Volterra-based model is infeasible. We therefore propose to reduce (3) to a memory polynomial structure [9]. In this structure, crossterms between a signal and terms of the same signal with different delays are not considered. For example, after reducing (3c) following the memory polynomial approach, only terms where $m_{1}=m_{2}=m_{3}$ are considered. Hence, pruning the Volterra series-based model to a memory polynomial structure results in

$$
\begin{aligned}
& b_{2 i}(n)= \\
& \left.\begin{array}{l}
\sum_{m_{1}=0}^{M_{1}} \sum_{p=0}^{\left(P_{1}-1\right) / 2} \alpha_{m_{1}}^{(2 p+1)} a_{1 i}\left(n-m_{1}\right) \\
\times\left|a_{1 i}\left(n-m_{1}\right)\right|^{2 p}
\end{array}\right\} \\
& +\sum_{m_{2}=0}^{M_{2}} \beta_{0 m_{2}}^{(1)} a_{2 i}\left(n-m_{2}\right) \\
& +\sum_{m_{3}=0}^{M_{3}} \sum_{m_{4}=0}^{M_{4}} \sum_{p=1}^{\left(P_{2}-1\right) / 2} \beta_{m_{4} m_{3}}^{(2 p+1)} \\
& \times a_{2 i}\left(n-m_{3}\right)\left|a_{1 i}\left(n-m_{4}\right)\right|^{2 p} \\
& \left.\begin{array}{l}
+\sum_{m_{5}=0}^{M_{5}} \sum_{m_{6}=0}^{M_{6}} \sum_{p=1}^{\left(P_{3}-1\right) / 2} \gamma_{m_{6} m_{5}}^{(2 p+1)} a_{2 i}^{*}\left(n-m_{5}\right) \\
\times\left(a_{1 i}\left(n-m_{6}\right)\right)^{p+1}\left(a_{1 i}^{*}\left(n-m_{6}\right)\right)^{p-1} .
\end{array}\right\}(4 \mathrm{c})
\end{aligned}
$$

In (4a), the terms containing only the signal $a_{1 i}(n)$ are combined. These terms describe the behavior of the PA due to the amplification of $a_{1 i}(n)$ and are the same as in a SISO memory polynomial model. In (4b) and (4c) the effects of coupling and mismatch and the mixing of these effects with PA nonlinearity

\footnotetext{
${ }^{1}$ Note that mismatch is also present in SISO TXs. The mismatch in SISO TXs is a function of the PA input $a_{1 i}(n)$, i.e. $a_{2 i}(n)$ is a function of $a_{1 i}(n)$. Hence, for a SISO TX with mismatch, (3) inherently reduces to a single-input model depending only on $a_{1 i}(n)$.
} 
are described, where in (4b) all terms containing $a_{2 i}(n)$ are combined, and in $(4 \mathrm{c})$ all terms containing its conjugate, i.e., $a_{2 i}^{*}(n)$, are combined. Note that the nonlinear orders $P_{1}, P_{2}$ and $P_{3}$, and the memory tap lengths $M_{1}, M_{2}, M_{3}, M_{4}, M_{5}$ and $M_{6}$ that are necessary to obtain a good model accuracy can be different from each other.

This reduced version of the model given in (3) has lower complexity, while still considering memory effects. Just as a single-input memory polynomial model, the model in (4) is linear in the coefficients. This means that the linear leastsquares method can be used for identification of the model coefficients from measurement data. However, for the dualinput model, two input signals, $a_{1 i}(n)$ and $a_{2 i}(n)$, as well as the output signal $b_{2 i}(n)$ need to be measured at the same time. A suitable measurement technique to obtain data for coefficient identification is presented in Section V-B1.

Note that while we chose to prune the full Volterra seriesbased model in (3) to a memory polynomial structure, any other pruning scheme, e.g., the generalized memory polynomial structure [17] or the dynamic deviation reduction-based pruning approach [18], can be applied just as well.

In order to compare the presented dual-input model to related research, it is worth mentioning a special case of the presented model, the quasi-static version. The model in (3) can be further pruned to a memoryless model, given by

$$
\begin{aligned}
& b_{2 i}(n)=a_{1 i}(n) \underbrace{\sum_{p=0}^{\left(P_{1}-1\right) / 2} \alpha^{(2 p+1)}\left|a_{1 i}(n)\right|^{2 p}}_{\bigwedge_{S_{21}}} \\
& +a_{2 i}(n) \underbrace{\left(\beta^{(1)}+\sum_{p=1}^{\left(P_{2}-1\right) / 2} \beta^{(2 p+1)}\left|a_{1 i}(n)\right|^{2 p}\right)}_{\bigwedge_{S_{22}}} \\
& +a_{2 i}^{*}(n) \underbrace{\sum_{p=1}^{\left(P_{3}-1\right) / 2} \gamma^{(2 p+1)}\left(a_{1 i}(n)\right)^{p+1}\left(a_{1 i}^{*}(n)\right)^{p-1}(5 \mathrm{c})}_{\bigwedge_{T_{22}}}
\end{aligned}
$$

where (5a) relates to $(4 a),(5 b)$ to $(4 b)$, and $(5 c)$ to $(4 c)$. As indicated by the braces in (5), this reduced version of the proposed model can be directly compared to the quasi-static PHD models [11], which, for the fundamental frequency, are described by

$$
\begin{aligned}
B_{21}(n)= & A_{1 i}(n) S_{21}\left(\left|A_{1 i}(n)\right|\right)+A_{2 i}(n) S_{22}\left(\left|A_{1 i}(n)\right|\right) \\
& +A_{2 i}^{*}(n) T_{22}\left(A_{1 i}(n)\right)
\end{aligned}
$$

where $A_{1 i}(n)$ and $A_{2 i}(n)$ are the two incident wave phasors, and $B_{21}(n)$ is the corresponding scattered wave. PHD models have been used to predict radiation patterns of active antenna arrays [4], [10]. However, since they are quasi-static and, as such, do not consider the history of the input signals, they are not suitable for wideband signals. As illustrated, the dynamic models proposed in (3) and (4) can therefore be seen as a generalization of the PHD models, to include memory effects.

\section{Prediction of Multi-Antenna TX Output}

Our goal is to predict the output signals $b_{2 i}(n)$ of a multiantenna TX, which are combined in $\mathbf{b}_{\mathbf{2}}(n)$. Each signal $a_{2 i}(n)$ is a function of the signals $\mathbf{b}_{\mathbf{2}}(n)$, as can be seen in (1). Hence, via the relation given in (1), both sides of the dual-input PA model in (4) contain current and past samples of the output signals $\mathbf{b}_{\mathbf{2}}(n)$. While the input signals $a_{1 i}(n)$ are known for all time samples, only past samples of the signals $\mathbf{b}_{\mathbf{2}}(n)$ can be known at the current time step $n$. Therefore, the output signals $\mathbf{b}_{\mathbf{2}}(n)$ can only be computed in a time-stepped manner, i.e. step by step for each sample time $n$ from $n=0$ to $N-1$. In this section, we first present such a time-stepped solution of the output at each antenna of the transmit array. We show how the results can be used to also compute the far-field radiation pattern of the full TX with minimum computational effort.

\section{A. Step-Wise Solution of Multi-Antenna TX Output}

In order to compute the samples of the output vector $\mathbf{b}_{\mathbf{2}}(n),(4)$ is transformed such that all current time samples of $\mathbf{b}_{\mathbf{2}}(n)$, i.e. the terms for $m=0$, are contained on one side of the equation. All past samples are combined on the other side together with all samples of $a_{1 i}(n)$. The detailed derivation is given in Appendix C. Then, (4) can be rewritten as

$$
\begin{aligned}
b_{2 i}(n) & =\left(\boldsymbol{\lambda}_{i}(0)\right)^{T} \mathbf{b}_{\mathbf{2}}(n) S_{22, i}\left(\left|a_{1 i}(\mathbf{n})\right|\right) \\
& +\left(\boldsymbol{\lambda}_{i}^{*}(0)\right)^{T} \mathbf{b}_{\mathbf{2}}{ }^{*}(n) T_{22, i}\left(a_{1 i}(\mathbf{n})\right) \\
& +f_{i}\left(a_{1 i}(\mathbf{n}), \mathbf{b}_{\mathbf{2}}\left(\mathbf{n}_{\text {past }}\right)\right)
\end{aligned}
$$

where $\mathbf{n}=\left[n, \ldots, n-\max \left(M_{1}, M_{4}, M_{6}\right)\right]^{T}, \mathbf{n}_{\text {past }}=[n-$ $\left.1, \ldots, n-\max \left(M_{2}, M_{3}, M_{5}\right)-K\right]^{T}$. Furthermore,

$$
\begin{aligned}
S_{22, i}\left(\left|a_{1 i}(\mathbf{n})\right|\right) & =\beta_{00}^{(1)}+\sum_{m_{4}=0}^{M_{4}} \sum_{p=1}^{\left(P_{2}-1\right) / 2} \beta_{m_{4} 0}^{(2 p+1)} \\
& \times\left|a_{1 i}\left(n-m_{4}\right)\right|^{2 p} \\
T_{22, i}\left(a_{1 i}(\mathbf{n})\right) & =\sum_{m_{6}=0}^{M_{6}} \sum_{p=1}^{\left(P_{3}-1\right) / 2} \gamma_{m_{6} 0}^{(2 p+1)} \\
& \times\left(a_{1 i}\left(n-m_{6}\right)\right)^{p+1}\left(a_{1 i}^{*}\left(n-m_{6}\right)\right)^{p-1} .
\end{aligned}
$$

The remaining terms of (4), i.e., all past samples of $\mathbf{b}_{\mathbf{2}}(n)$ and all samples of $a_{1 i}(n)$ are contained by

$$
\begin{aligned}
f_{i}\left(a_{1 i}(\mathbf{n}), \mathbf{b}_{\mathbf{2}}\left(\mathbf{n}_{\text {past }}\right)\right)= \\
\quad \sum_{m_{1}=0}^{M_{1}} \sum_{p=0}^{\left(P_{1}-1\right) / 2} \alpha_{m_{1}}^{(2 p+1)} a_{1 i}\left(n-m_{1}\right)\left|a_{1 i}\left(n-m_{1}\right)\right|^{2 p} \\
+\sum_{k=1}^{K}\left(\boldsymbol{\lambda}_{i}(k)\right)^{T} \mathbf{b}_{\mathbf{2}}(n-k) \\
\quad \times\left(\beta_{00}^{(1)}+\sum_{m_{4}=0}^{M_{4}} \sum_{p=1}^{\left(P_{2}-1\right) / 2} \beta_{m_{4} 0}^{(2 p+1)}\left|a_{1 i}\left(n-m_{4}\right)\right|^{2 p}\right) \\
+\sum_{m_{2}=1}^{M_{2}} \beta_{0}^{(1)} \sum_{m_{2}}^{K}\left(\boldsymbol{\lambda}_{i}(k)\right)^{T} \mathbf{b}_{\mathbf{2}}\left(n-k-m_{2}\right) \\
+\sum_{m_{3}=1}^{M_{3}} \sum_{m_{4}=0}^{M_{4}} \sum_{p=1}^{\left(P_{2}-1\right) / 2} \beta_{m_{4} m_{3}}^{(2 p+1)}\left|a_{1 i}\left(n-m_{4}\right)\right|^{2 p}
\end{aligned}
$$




$$
\begin{aligned}
& {\left[\begin{array}{l}
\Re\left\{\mathbf{b}_{\mathbf{2}}(n)\right\} \\
\Im\left\{\mathbf{b}_{\mathbf{2}}(n)\right\}
\end{array}\right]=\left[\begin{array}{cc}
\mathbf{I}-\Re\left\{\mathbf{S}_{\mathbf{2 2}}\left(\left|\mathbf{a}_{\mathbf{1}}(\mathbf{n})\right|\right) \boldsymbol{\Lambda}(0)\right\}-\Re\left\{\mathbf{T}_{\mathbf{2 2}}\left(\mathbf{a}_{\mathbf{1}}(\mathbf{n})\right) \boldsymbol{\Lambda}^{*}(0)\right\} & \Im\left\{\mathbf{S}_{\mathbf{2 2}}\left(\left|\mathbf{a}_{1}(\mathbf{n})\right|\right) \boldsymbol{\Lambda}(0)\right\}-\Im\left\{\mathbf{T}_{\mathbf{2 2}}\left(\mathbf{a}_{\mathbf{1}}(\mathbf{n})\right) \boldsymbol{\Lambda}^{*}(0)\right\} \\
-\Im\left\{\mathbf{S}_{\mathbf{2 2}}\left(\left|\mathbf{a}_{\mathbf{1}}(\mathbf{n})\right|\right) \boldsymbol{\Lambda}(0)\right\}-\Im\left\{\mathbf{T}_{\mathbf{2 2}}\left(\mathbf{a}_{\mathbf{1}}(\mathbf{n})\right) \boldsymbol{\Lambda}^{*}(0)\right\} & \mathbf{I}-\Re\left\{\mathbf{S}_{\mathbf{2 2}}\left(\left|\mathbf{a}_{\mathbf{1}}(\mathbf{n})\right|\right) \boldsymbol{\Lambda}(0)\right\}+\Re\left\{\mathbf{T}_{\mathbf{2 2}}\left(\mathbf{a}_{\mathbf{1}}(\mathbf{n})\right) \boldsymbol{\Lambda}^{*}(0)\right\}
\end{array}\right]^{-1}} \\
& \times\left[\begin{array}{l}
\Re\left\{\mathbf{f}\left(\mathbf{a}_{1}(\mathbf{n}), \mathbf{b}_{\mathbf{2}}\left(\mathbf{n}_{\text {past }}\right)\right)\right\} \\
\Im\left\{\mathbf{f}\left(\mathbf{a}_{\mathbf{1}}(\mathbf{n}), \mathbf{b}_{\mathbf{2}}\left(\mathbf{n}_{\text {past }}\right)\right)\right\}
\end{array}\right]
\end{aligned}
$$

$$
\begin{aligned}
& \times \sum_{k=0}^{K}\left(\boldsymbol{\lambda}_{i}(k)\right)^{T} \mathbf{b}_{\mathbf{2}}\left(n-k-m_{3}\right) \\
& +\sum_{m_{6}=0}^{M_{6}} \sum_{p=1}^{\left(P_{3}-1\right) / 2} \gamma_{m_{6} 0}^{(2 p+1)}\left(a_{1 i}\left(n-m_{6}\right)\right)^{p+1}\left(a_{1 i}^{*}\left(n-m_{6}\right)\right)^{p-1} \\
& \times \sum_{k=1}^{K}\left(\boldsymbol{\lambda}_{i}^{*}(k)\right)^{T} \mathbf{b}_{\mathbf{2}}^{*}(n-k) \\
& +\sum_{m_{5}=1}^{M_{5}} \sum_{m_{6}=0}^{M_{6}} \sum_{p=1}^{\left(P_{3}-1\right) / 2} \gamma_{m_{6} m_{5}}^{(2 p+1)} \\
& \times\left(a_{1 i}\left(n-m_{6}\right)\right)^{p+1}\left(a_{1 i}^{*}\left(n-m_{6}\right)\right)^{p-1} \\
& \times \sum_{k=0}^{K}\left(\boldsymbol{\lambda}_{i}^{*}(k)\right)^{T} \mathbf{b}_{\mathbf{2}}^{*}\left(n-k-m_{5}\right) .
\end{aligned}
$$

All transmit paths of the TX described separately by (7) are combined to obtain the output of all TX antennas in

$$
\begin{aligned}
\mathbf{b}_{\mathbf{2}}(n) & =\mathbf{S}_{\mathbf{2 2}}\left(\left|\mathbf{a}_{\mathbf{1}}(\mathbf{n})\right|\right) \boldsymbol{\Lambda}(0) \mathbf{b}_{\mathbf{2}}(n) \\
& +\mathbf{T}_{\mathbf{2 2}}\left(\mathbf{a}_{\mathbf{1}}(\mathbf{n})\right) \boldsymbol{\Lambda}^{*}(0) \mathbf{b}_{\mathbf{2}}^{*}(n)+\mathbf{f}\left(\mathbf{a}_{\mathbf{1}}(\mathbf{n}), \mathbf{b}_{\mathbf{2}}\left(\mathbf{n}_{\text {past }}\right)\right)(11)
\end{aligned}
$$

with

$\mathbf{S}_{\mathbf{2 2}}\left(\left|\mathbf{a}_{\mathbf{1}}(\mathbf{n})\right|\right)=\operatorname{diag}\left\{S_{22,1}\left(\left|a_{11}(\mathbf{n})\right|\right), \ldots, S_{22, L}\left(\left|a_{1 L}(\mathbf{n})\right|\right)\right\}$ $\mathbf{T}_{\mathbf{2 2}}\left(\mathbf{a}_{\mathbf{1}}(\mathbf{n})\right)=\operatorname{diag}\left\{T_{22,1}\left(a_{11}(\mathbf{n})\right), \ldots, T_{22, L}\left(a_{1 L}(\mathbf{n})\right)\right\}$ $\mathbf{f}\left(\mathbf{a}_{\mathbf{1}}(\mathbf{n}), \mathbf{b}_{\mathbf{2}}\left(\mathbf{n}_{\text {past }}\right)\right)=$

$$
\left[f_{1}\left(a_{11}(\mathbf{n}), \mathbf{b}_{\mathbf{2}}\left(\mathbf{n}_{\text {past }}\right)\right), \ldots, f_{L}\left(a_{1 L}(\mathbf{n}), \mathbf{b}_{\mathbf{2}}\left(\mathbf{n}_{\text {past }}\right)\right)\right]^{T}
$$

$\boldsymbol{\Lambda}(0)=\left[\boldsymbol{\lambda}_{1}(0), \ldots, \boldsymbol{\lambda}_{L}(0)\right]^{T}$.

In (11), all currently unknown samples of the output signals are included in $\mathbf{b}_{\mathbf{2}}(n)$, while all past and known signal samples are contained in $\mathbf{f}\left(\mathbf{a}_{\mathbf{1}}(\mathbf{n}), \mathbf{b}_{\mathbf{2}}\left(\mathbf{n}_{\text {past }}\right)\right)$. Equation (11) can be solved analytically for $\mathbf{b}_{\mathbf{2}}(n)$. First, (11) is split into real and imaginary parts, denoted by $\Re\{\cdot\}$ and $\Im\{\cdot\}$. The real and imaginary part of the output $\mathbf{b}_{\mathbf{2}}(n)$ are obtained by (13), and $\mathbf{b}_{\mathbf{2}}(n)$ is computed by $\mathbf{b}_{\mathbf{2}}(n)=\Re\left\{\mathbf{b}_{\mathbf{2}}(n)\right\}+j \Im\left\{\mathbf{b}_{\mathbf{2}}(n)\right\}$.

The solution presented in (13) allows the output of all TX paths to be determined for any input signal combination step by step for each time sample $n$. Since the mutual coupling is usually dominated by neighboring antenna elements, the problem is sparse and well-conditioned. Well established numerical techniques can therefore be employed to enable simulations of very large multi-antenna TX systems with limited computational effort.

\section{B. Prediction of TX Radiation Pattern}

By including knowledge about the antenna element radiation patterns, it is possible to predict the total far-field electromagnetic (EM) wave generated by the TX. In most commercial EM software, the single antenna embedded farfield data is conveniently available by post-processing of the same simulation results that are used to compute the antenna characteristics. Using this data, the total far-field EM wave $E_{\text {tot }}(\theta, \phi)$ is calculated as the superposition of the single antenna output signals $b_{2 i}(n)$, scaled by the far-field radiation pattern $E_{i}(\theta, \phi)$ of the corresponding antenna element. This is given by

$$
E_{\text {tot }}(\theta, \phi, n)=\left(\mathbf{b}_{\mathbf{2}}(n)\right)^{T} \mathbf{E}(\theta, \phi)
$$

where $\mathbf{E}(\theta, \phi)$ is an $L \times 1$ vector containing the unity amplitude far-field pattern of each antenna element with all other elements terminated with the reference impedance $(50 \mathrm{Ohm})$.

Even though the far-field is not experimentally evaluated in Section VI, the theory opens up several interesting possibilities to study how the radiation pattern and far-field distortion is influenced by interactions between PAs and antennas. As an example, we have used it to investigate far-field distortion effects in phased-array transmitters [19].

\section{Implementation of the Simulation Technique}

In order to use the presented theory to predict the output of a multi-antenna TX, several steps are necessary. First, the individual components of the TX, i.e., PAs, antenna elements and array configuration, have to be chosen or designed. Then, the PA model coefficients and the antenna array characteristics have to be identified. The identification is done through individual measurements of the components, or from circuit/antenna simulation. For example, the antenna array characteristics can be identified from measurements of the array scattering parameters versus frequency. The PA model coefficients can be identified using active loadpull measurements [20], [21]. All identified coefficients can then be used in (13) to predict the output of the multi-antenna TX for kown input signals.

Using separate, rather simple measurements or simulations for the characterization of the different components, the system performance can therefore be evaluated for different types of components without great effort. An implementation example for a four-path TX, including all identification procedures, is explained in detail in the following section.

\section{Mimo System-BAsed TX Demonstrator Design}

In the remainder of this paper, the theory presented in the previous sections is evaluated using a wireless multi-antenna TX, where each TX path is driven by different, independent input signals. Operating a TX in such a way is commonly done 


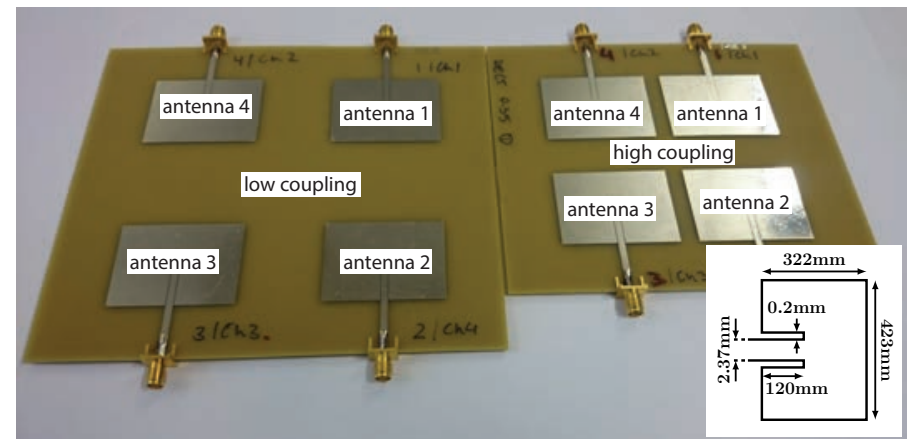

Fig. 2. Photo of the antenna arrays, and the dimensions of an antenna element.

in wireless communications-based MIMO systems. A fourelement TX demonstrator was designed for simulations and measurements.

In this section, we explain the design and characterization of the two main components that are necessary to build such a TX, i.e., the TX antenna array and the PAs. The measurement and simulation results are later presented and compared in Section VI.

\section{A. Antenna Design}

A microstrip patch antenna is selected as radiation element of the antenna arrays. Two rectangular four-element antenna arrays were designed to be able to observe different coupling intensities. In Fig. 2, a photograph of the arrays is shown, where also the dimensions of a single antenna element are given. For the array with higher coupling, distance between antenna element centers is $49 \mathrm{~mm}$, and for the array with lower coupling, the distance is $70 \mathrm{~mm}$. The antenna arrays were designed using the Keysight Momentum EM simulator for a resonant frequency of $f_{c}=2.14 \mathrm{GHz}$, and manufactured using FR4 substrate $\left(\epsilon_{r}=4.4, \tan \delta=0.02\right.$, and thickness $=62 \mathrm{mil}$ $(1.57 \mathrm{~mm}))$.

The characteristics of the manufactured arrays were determined in measurements with a two-port vector network analyzer (VNA) using pairwise measurements with the other ports terminated in $50 \mathrm{Ohm}$. The measured array scattering parameters $\mathbf{S}_{\text {ant }}$ versus frequency are shown in Fig. 3. The array scattering parameters are shown for only one antenna of each array, since they are similar for each of the antennas due to reciprocity. The resonant frequency of the antenna elements was measured around $f_{c}=2.12 \mathrm{GHz}$, as opposed to the targeted $2.14 \mathrm{GHz}$. This is most likely due to small deviations of the actual substrate characteristics from the characteristics provided by the manufacturer which were used in the design process. However, a small change in antenna resonant frequency is not critical to our experiments. All subsequent measurements and simulations are therefore referring to a center frequency of $f_{c}=2.12 \mathrm{GHz}$. The highest coupling factor is between two directly adjacent antenna elements, and it was measured as around $-12 \mathrm{~dB}$ for the high-coupling array, and around $-24 \mathrm{~dB}$ for the low-coupling array. In order to use the measured antenna characteristics in

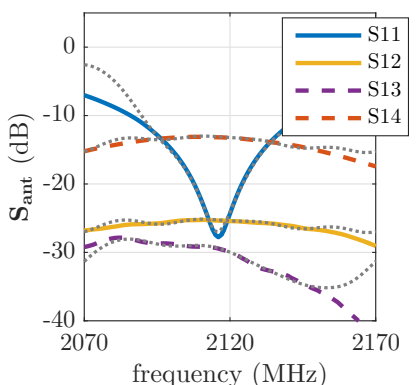

(a)

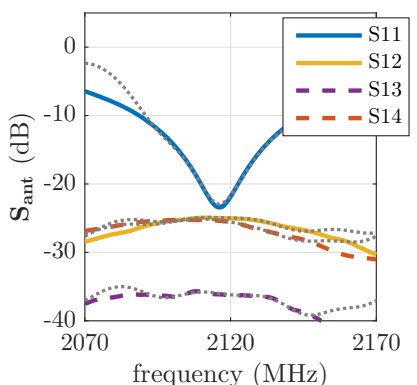

(b)
Fig. 3. Antenna array characteristics $\mathbf{S}_{\text {ant }}$ versus frequency for antenna 1 for (a) high-coupling array, and (b) low-coupling array. The figures show the scattering parameters for: reflection (S11), adjacent element (S14), opposite element (S12), and diagonally opposite element (S13). The results for the corresponding extracted FIR filters with 5 filter taps are shown in dotted gray.

the simulations, the single-frequency S-parameters at center frequency $f_{c}=2.12 \mathrm{GHz}$, as well as FIR filters describing the antenna characteristics were extracted from the measured array scattering parameters. The FIR filters were designed using linear least-squares estimation. For each FIR filter, a test signal $x(n)$ was filtered in frequency domain using the corresponding measured S-parameters $\mathbf{S}_{\mathbf{a n t}}$, resulting in a signal $y(n)$. The FIR filter coefficients $\boldsymbol{\lambda}=[\lambda(0), \ldots, \lambda(K)]^{T}$ were found in time domain as the least-squares estimate

$$
\boldsymbol{\lambda}=\mathbf{X}^{+} \mathbf{y}
$$

where the pseudoinverse $\mathbf{X}^{+}=\left(\mathbf{X}^{H} \mathbf{X}\right)^{-1} \mathbf{X}^{H}$ with $\mathbf{X}=$ $\left[\mathbf{x}_{0}, \ldots, \mathbf{x}_{K}\right]$ and $\mathbf{x}_{k}=[x(0-k), \ldots, x(N-1-k)]^{T}$, and $\mathbf{y}=[y(0), \ldots, y(N-1)]^{T}$. The demonstrator is designed for an input signal bandwidth of $20 \mathrm{MHz}$. The bandwidth of the test signal $x(n)$ was $60 \mathrm{MHz}$, resulting in an accurate FIR filter description over the same bandwidth, which includes also the first side-bands. The results for five-tap filters, i.e. $K=4$, are shown together with the measured S-parameters in Fig. 3.

\section{B. PA Characterization and Modeling}

The demonstrator is based on four GaAs PA evaluation boards from Skyworks with identical design (SKY66001-11). The frequency range of the PAs is $2.1-2.2 \mathrm{GHz}$. The PAs have integrated couplers at their outputs. Each PA in the demonstrator was supplied with $3.3 \mathrm{~V}$.

In order to model and predict the output of the multi-antenna TX in simulations, the coefficients $\alpha, \beta$ and $\gamma$ of the dual-input PA model described in (4) must be identified.

1) Active Load-Pull Measurements: In order to characterize dual-input PAs as in a multi-antenna TX, it is necessary to synchronously inject and measure signals both at the PA input and output at well-defined reference planes [20]. Hence, a mixed-mode active load-pull measurement setup [21] is used for the experimental extraction of the dual-input PA model coefficients. The active load-pull setup is calibrated using a short-open-load-through (SOLT) technique in a similar way as it is done with VNAs. Additionally, power and phase reference calibration is performed using techniques described in [22]. A 


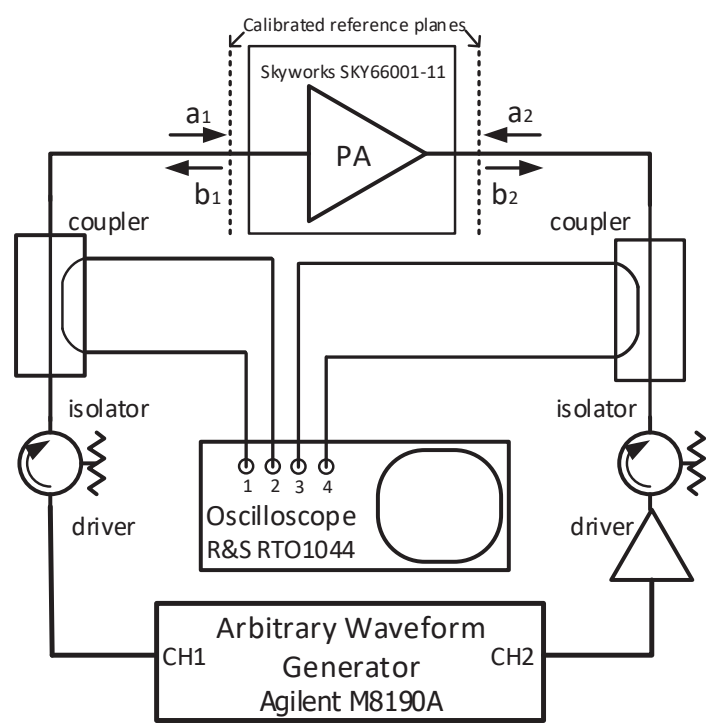

Fig. 4. Block diagram of the active load-pull measurement system that is used to extract the model coefficients to characterize the PAs.

block diagram of the measurement setup is shown in Fig. 4. This setup allows the injection of different multi-sine signals at the PA input and output, respectively. The incident and reflected waves at the PA input/output calibrated reference plane, i.e., $a_{1}(n), b_{1}(n), a_{2}(n)$ and $b_{2}(n)$, can be accurately measured. Hence, this measurement system can be used to emulate a dual-input PA fitting the described model. The model coefficients can be extracted from the measured data following the process explained in Appendix B.

2) Results of Model Coefficient Extraction: Each of the four PAs was measured and characterized individually. Measurements were taken for an input signal bandwidth of $B_{a}=20 \mathrm{MHz}$ for both $a_{1}(n)$ and $a_{2}(n)$, where the calibration and measurement bandwidth was set to $f_{s}=5 B_{a}=100$ $\mathrm{MHz}$ to allow capturing nonlinear effects in sidebands. Both $a_{1}(n)$ and $a_{2}(n)$ were multi-sine signals with randomly chosen phases and an amplitude probability density function that matched that of the signals which are later used in the multiantenna TX demonstrator in Section VI. The average power of the input signal $a_{1}(n)$ was set to $-7 \mathrm{dBm}$ and the average power of the signal $a_{2}(n)$ was chosen to emulate a coupling factor of $-12 \mathrm{~dB}$ between antennas. Using the measured data for $a_{1}(n), a_{2}(n)$ and $b_{2}(n)$ and (4), the least-squares method was used to extract model coefficients for each of the four PAs.

The accuracy of the model is evaluated using the normalized mean square error (NMSE) and the adjacent channel error power ratio (ACEPR) [23]. The NMSE between the model output $b_{\text {mod }}(n)$ and the measured data $b_{\text {meas }}(n)$ is calculated as

$$
\mathrm{NMSE}=\frac{\sum_{n=0}^{N-1}\left|b_{\text {meas }}(n)-b_{\text {mod }}(n)\right|^{2}}{\sum_{n=0}^{N-1}\left|b_{\text {meas }}(n)\right|^{2}}
$$

with $N$ being the total number of time samples. The ACEPR

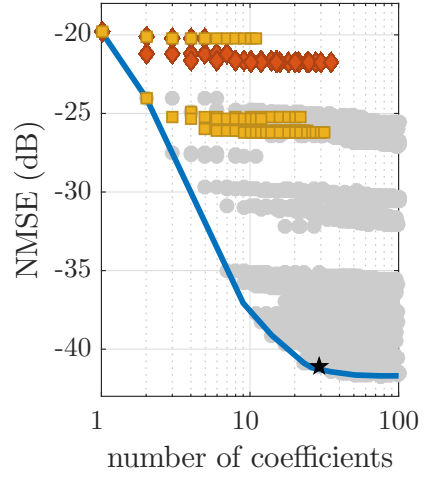

(a)

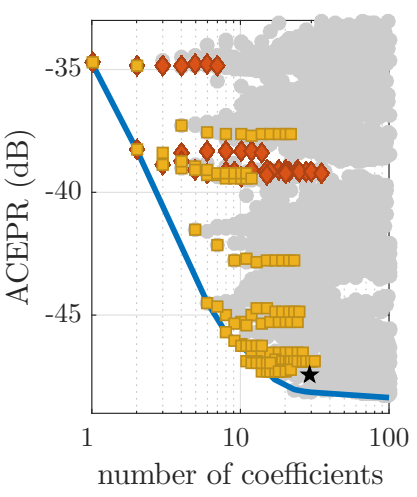

(b)
Fig. 5. (a) NMSE versus number of coefficients. (b) ACEPR versus number of coefficients. The results are for one of the PAs (PA 1). Each light gray dot indicates a separate combination of model parameters $P_{1}, P_{2}, P_{3}, M_{1}, M_{2}, M_{3}, M_{4}, M_{5}$ and $M_{6}$ in (4). The diamonds indicate results for a single-input model, and the squares results for a quasi-static model. The solid lines indicate the lowest achievable NMSE and ACEPR for a specific number of coefficients. The stars indicate the result given in Table I where the NMSE is $-43.9 \mathrm{~dB}$ for 29 coefficients with $P_{1}=9, P_{2}=$ $P_{3}=5, M_{1}=2, M_{2}=M_{3}=M_{4}=M_{6}=1$, and $M_{5}=0$, and the corresponding ACEPR is $-49.8 \mathrm{~dB}$.

TABLE I

PA IDENTIFICATION RESULTS FOR DIFFERENT PA MODELS.

\begin{tabular}{|c|c|c|c|c|c|}
\hline & PA & $\begin{array}{l}\text { NMSE } \\
(\mathrm{dB})\end{array}$ & $\begin{array}{c}\text { ACEPR } \\
(\mathrm{dB})\end{array}$ & \#coeff & $\begin{array}{c}\left\{P_{1}, P_{2}, P_{3}, M_{1}, M_{2},\right. \\
\left.M_{3}, M_{4}, M_{5}, M_{6}\right\}\end{array}$ \\
\hline \multirow{4}{*}{$\begin{array}{l}\text { Dू } \\
0 \\
0 \\
0 \\
0 \\
0\end{array}$} & 1 & -41.1 & -47.5 & 29 & $\{9,5,5,2,1,1,1,0,1\}$ \\
\hline & 2 & -40.2 & -46.5 & 26 & $\{9,7,7,2,1,1,0,0,0\}$ \\
\hline & 3 & -40.5 & -47.2 & 35 & $\{9,7,7,2,1,1,1,0,1\}$ \\
\hline & 4 & -40.4 & -47.1 & 35 & $\{9,7,7,2,1,1,1,0,1\}$ \\
\hline \multirow{4}{*}{ 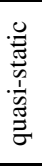 } & 1 & -26.2 & -46.6 & 14 & $\{9,9,9,0,0,0,0,0,0\}$ \\
\hline & 2 & -26.0 & -45.7 & 14 & $\{9,9,9,0,0,0,0,0,0\}$ \\
\hline & 3 & -26.0 & -45.7 & 14 & $\{9,9,9,0,0,0,0,0,0\}$ \\
\hline & 4 & -25.9 & -45.5 & 14 & $\{9,9,9,0,0,0,0,0,0\}$ \\
\hline \multirow{4}{*}{ 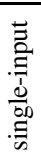 } & 1 & -21.7 & -39.2 & 30 & $\{9,0,0,5,0,0,0,0,0\}$ \\
\hline & 2 & -22.7 & -38.8 & 30 & $\{9,0,0,5,0,0,0,0,0\}$ \\
\hline & 3 & -23.7 & -38.0 & 30 & $\{9,0,0,5,0,0,0,0,0\}$ \\
\hline & 4 & -24.2 & -37.7 & 30 & $\{9,0,0,5,0,0,0,0,0\}$ \\
\hline
\end{tabular}

is calculated as

$$
\mathrm{ACEPR}=\frac{\sum_{f_{a d j}}\left|B_{\text {meas }}(f)-B_{\text {mod }}(f)\right|^{2}}{\sum_{f_{c h}}\left|B_{\text {meas }}(f)\right|^{2}}
$$

where $B_{\text {mod }}(f)$ and $B_{\text {meas }}(f)$ are the Fourier transform of the model output and the measured data, $f_{c h}$ denotes inband frequencies and $f_{a d j}$ frequencies in the adjacent channel. The ACEPR is calculated separately for both the upper and the lower adjacent channels, with the maximum used for evaluation.

Model coefficients were extracted for various combinations of parameters $P_{1}, P_{2}, P_{3}, M_{1}, M_{2}, M_{3}, M_{4}, M_{5}$ and $M_{6}$. Fig. 5(a) shows an example of the resulting NMSEs, and Fig. 5(b) an example of the resulting ACEPRs for different numbers of coefficients for one of the PAs. The figure also indicates the NMSEs and ACEPRs obtained for a single-input PA model, i.e. a model where $a_{2}(n)$ is not considered, and 
for a quasi-static model, i.e. $M_{1}=M_{2}=M_{3}=M_{4}=M_{5}=$ $M_{6}=0$. As can be seen from the figure, the proposed PA model outperforms quasi-static and single-input PA models in terms of NMSE. The ACEPR for the proposed model and the quasi-static model are very similar, while the single-input model performs worse. The figure also shows that there is a trade-off between the number of coefficients, i.e., complexity, and model accuracy. Hence, for each PA, a parameter combination that leads to a low number of coefficients while achieving satisfying results for both NMSE and ACEPR has to be found. The chosen parameter combinations for the four PAs and the resulting NMSEs and ACEPRs are given in Table I. Results are given for the proposed PA model as well as a single-input PA model and a quasi-static model.

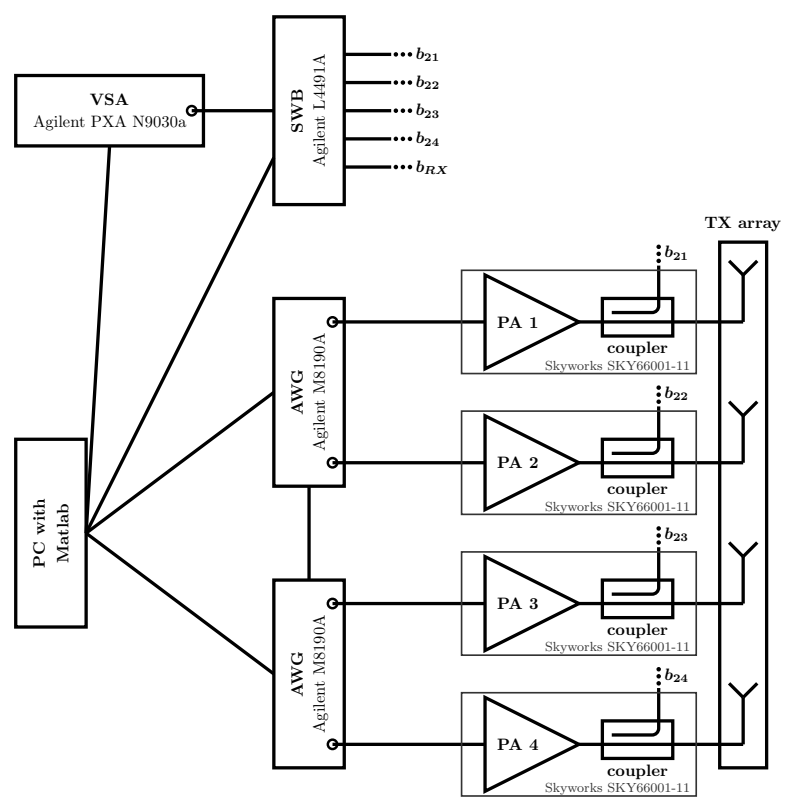

Fig. 6. Block diagram of the setup for the evaluation of the four-element TX demonstrator.

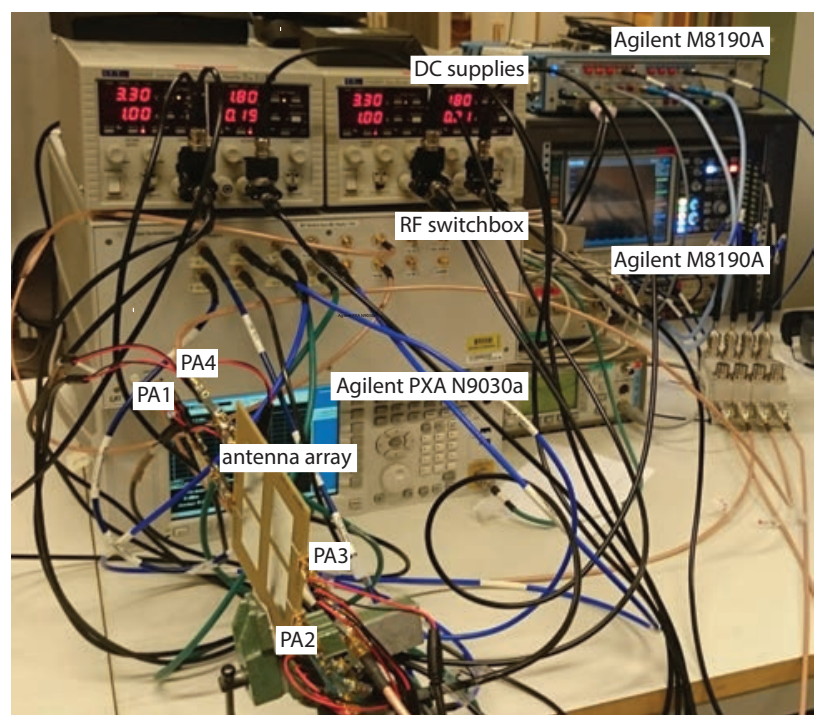

Fig. 7. Photo of the measurement setup in the lab.

\section{RESULTS}

In this section, the simulation results of the proposed technique are validated against measurement results of the fourelement TX for the array with smallest antenna separation, i.e. the highest coupling. We then show that our technique can be used to predict the amount of distortion introduced by crosstalk and mismatch separately from the distortion introduced by amplification of $a_{1 i}(n)$. Finally, measurements were performed to investigate whether conventional SISO DPD is a sufficient linearization technique, or if dedicated multi-antenna TX DPD, often called MIMO DPD, is necessary.

\section{A. Validation for High-Coupling Four-Element Array}

A block diagram of the measurement setup of the fourelement TX is shown in Fig. 6, and a photo of the setup in the lab is shown in Fig. 7. Four different and independent orthogonal frequency-division multiplexing (OFDM) signals with bandwidths $B_{a}=20 \mathrm{MHz}$ and peak-to-average power ratios of around $8.5 \mathrm{~dB}$ were generated in MATLAB. Two synchronized high-speed dual-channel arbitrary waveform generators (AWG, Agilent M8190A) synthesized the four driving signals for the PAs. The integrated couplers of the PA testboards were used to measure the individual PA output signals. The manufactured four-element antenna arrays were used as TX array. The individual PA output signals were connected to an RF switchbox with multiple inputs and one output. Only one signal at a time was switched through to the output of the switchbox, which was connected to a vector signal analyzer (VSA, Agilent PXA N9030a). This way, each signal was individually captured by the VSA. Processing was done in MATLAB.

For the simulations, the same four OFDM signals as in the measurements were used. Simulations were performed for the following techniques and settings:

- the proposed technique using the proposed dynamic PA models, given in (4), in combination with a singlefrequency S-parameter description of the antenna array, given in (2)

- the proposed technique using dynamic PA models, given in (4), in combination with a five-tap filter description of the antenna array derived from multi-frequency Sparameters, given in (1)

- the proposed technique using the quasi-static PA models, given in (5), in combination with a single-frequency $\mathrm{S}$ parameter description of the antenna array, given in (2)

- conventional single-input PA models for each transmit path.

A comparison of the spectrum of each individual simulated PA output to the spectrum of the respective measured PA output is shown in Fig. 8 for the proposed technique using the dynamic PA models: Fig. 8(a) shows the simulation results for a single-frequency S-parameters description, and Fig. 8(b) the simulation results for a filter description of the antenna array. The error spectra are also shown. As can be seen in the figures, the simulated spectra match well with the measured spectra for all presented cases. Fig. 9 shows the normalized error spectra. The error spectra are shown for the proposed technique using 


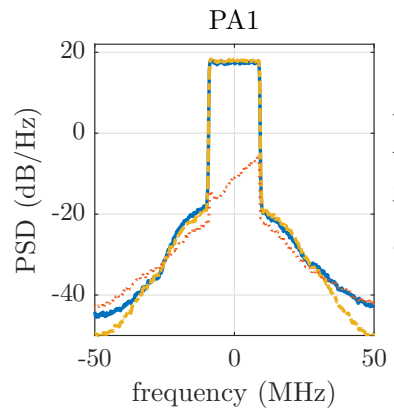

PA3
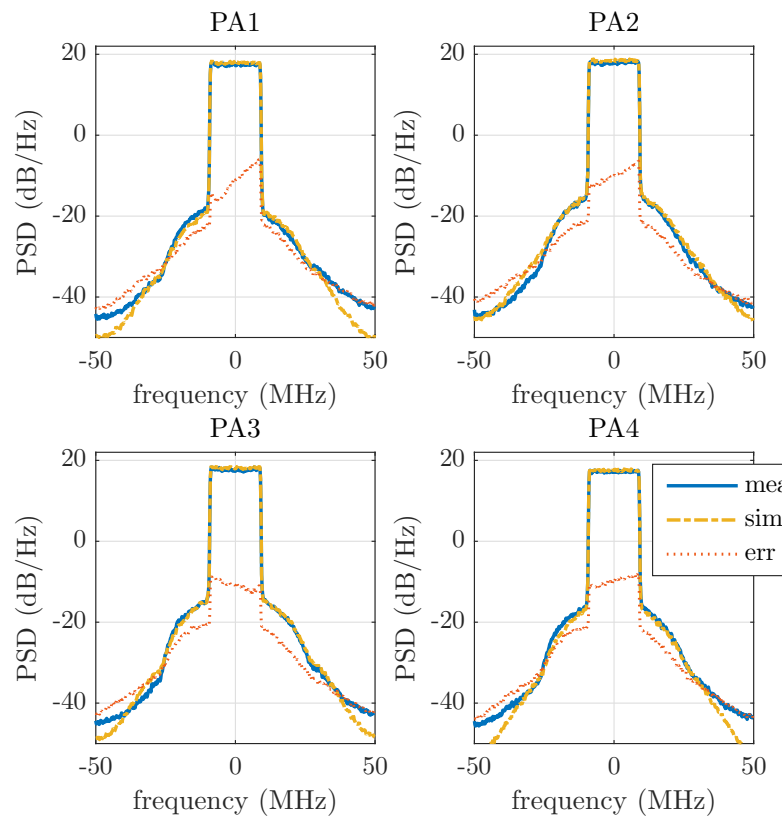

PA4

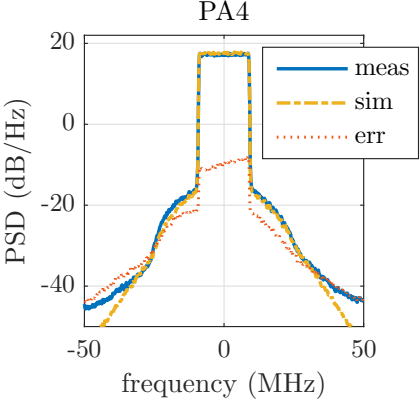

(a)
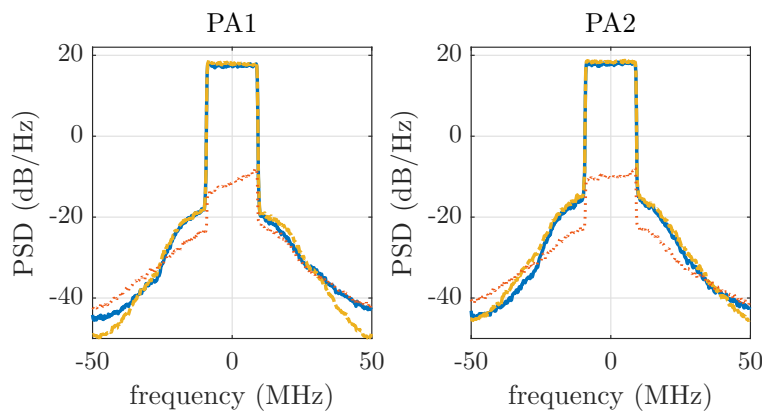

PA3
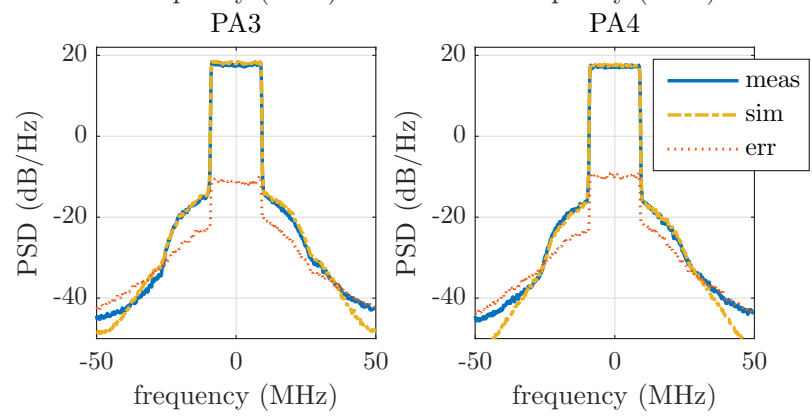

(b)

Fig. 8. PA output spectra of measurement (meas), simulation with the proposed technique (sim), and error (err) for: (a) dynamic PA models and single-frequency $\mathrm{S}$-parameters, and (b) dynamic PA models and filters derived from multi-frequency S-parameters.

dynamic PA models, as well as quasi-static PA models. The error spectra for simulations using single-input PA models are given as well. It can clearly be noticed that the singleinput PA model performs significantly worse than the proposed technique in all cases. It seems that the performance of the proposed technique is slightly better when using the dynamic PA models with an FIR antenna description performs than when using quasi-static PA models and dynamic PA models with a single-frequency S-parameters antenna description.

To give a more exact measure of performance, the NMSEs and ACEPRs for all simulations are given in Fig. 10. In all cases, the best performance in terms of ACEPR was obtained with the proposed technique with the dynamic PA models in combination with an antenna filter description, followed by the dynamic PA models in combination with an antenna single-frequency S-parameter description, and the quasi-static PA models. With one exception (PA3), the same is true for the performance in terms of NMSE. It is clear that the simulation using single-input PA models is not suitable to predict the output of the four-element transmitter. The proposed technique with quasi-static PA models performs slightly worse than with the proposed dynamic models. This is because of the dynamic effects of the PA and antenna that are not considered in the quasi-static PA models. The best overall performance of the the proposed technique using the dynamic PA models with the antenna filter description can be explained when looking at the measurements of the antenna scattering parameters versus frequency in Fig. 3, where it can be seen that the coupling between the antennas is not perfectly frequency flat within the measurement bandwidth. In addition to that, the reflection shows rather strong frequency dependent behavior, though it contributes only little power compared to the coupling from adjacent antennas. For these reasons, the single-frequency S-parameter description does not sufficiently describe the behavior of the antenna array, and the filter description leads to a slightly better result.

Overall, it should be noted that the accuracy of our evaluation is limited by several factors. The model coefficients and parameters are extracted from different, separate measurement setups rather than the setup of the full TX. Ideally, behavioral model extraction is done in the exact same setup and environment as the evaluation measurements, since every difference in the conditions, e.g. measurement instrument imprecision, cables, temperature, etc. can cause small uncertainties that in combination influence the outcome of the result. However, performing such measurements requires the full implementation of the TX. Building a full multi-antenna TX for large antenna arrays is very costly and time consuming. In addition to that, the measurements that are necessary to extract models from a full multi-antenna TX are extremely complicated, difficult to calibrate and synchronize, and require expensive equipment which is often not available. In fact, one of the benefits of our technique is that it enables the prediction of the output of a multi-antenna TX by measuring its individual components, and by doing so getting an estimate of the performance without the need to implement the whole TX. This allows for investigating many design options, and making design changes in early development phases, and gives insights into the nonlinear interactions between circuits, antennas and signals.

\section{B. Analysis of Distortion Due to Crosstalk and Mismatch}

An interesting application of our work is the possibility to investigate mismatch and crosstalk effects in multi-antenna 

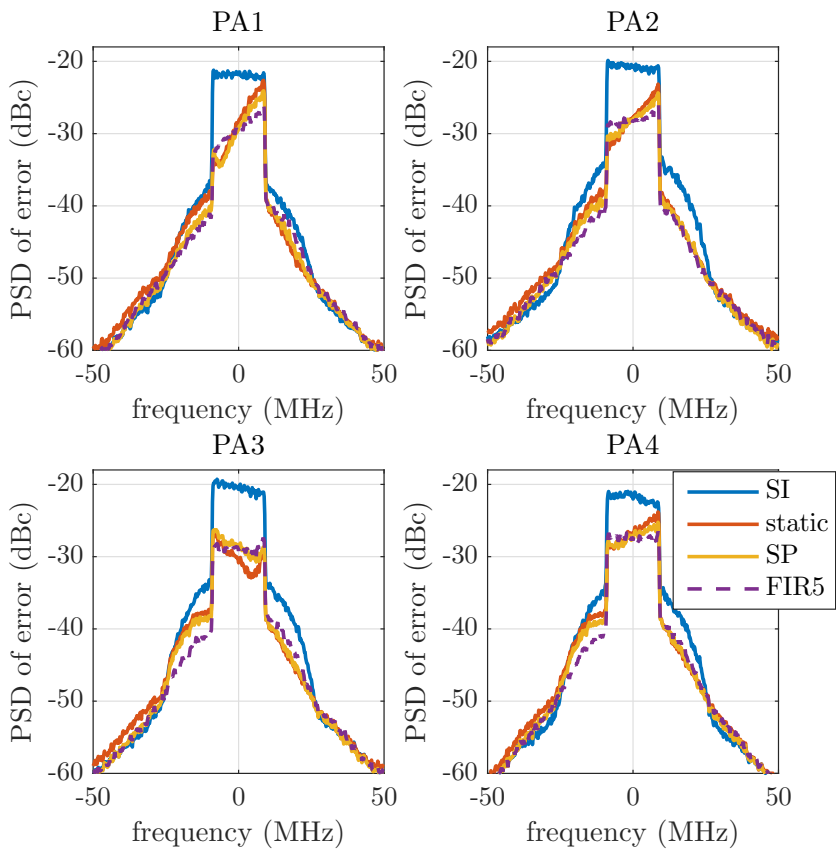

Fig. 9. Error spectra of PA output. The plot shows the errors for: the proposed technique with proposed dynamic PA models and single-frequency S-Parameter antenna array description (SP), the proposed technique with dynamic PA models and filter antenna array description (FIR5), the proposed technique with quasi-static PA models and single-frequency S-Parameter antenna array description (static), and simulations with single-input PA models (SI).

systems for different antenna arrays. For this purpose, we want to be able to observe these effects separately from the effects that are introduced by the nonlinear amplification of $a_{1 i}(n)$ in the PA. Investigating only the effects specific to multi-antenna enables a convenient comparison of the crosstalk effects for different antenna arrays. It can also give an idea about the amount of additional distortion that has to be expected in a multi-antenna TX as compared to a SISO TX. It will therefore help determine if it is necessary to design advanced DPD techniques, or if conventional SISO DPD can be enough to reach desired signal quality requirements such as adjacent channel power ratio (ACPR) even in a multi-antenna TX.

By compensating for the effects that are introduced during amplification of $a_{1 i}(n)$, our technique can be used to investigate only mismatch and crosstalk effects. To this end, a SISO DPD was identified and applied in both measurements and simulations. SISO DPDs for each of the paths were designed separately. This was done by driving each PA in a single-path scenario, i.e. by applying a signal to only one path of the TX, while for the other path the signal was set to zero, where in the measurements biasing was on for both amplifiers. A vector switched DPD as proposed in [24] was used. The obtained SISO DPD will only compensate for distortion caused by amplification of $a_{1 i}(n)$, while not eliminating the crosstalk and mismatch effects. Hence, the remaining out-of-band distortion is due to crosstalk and mismatch effects.

Fig. 11 shows the measured and simulated spectra of the PA output of TX path 1. In Fig. 11(a), the results for the
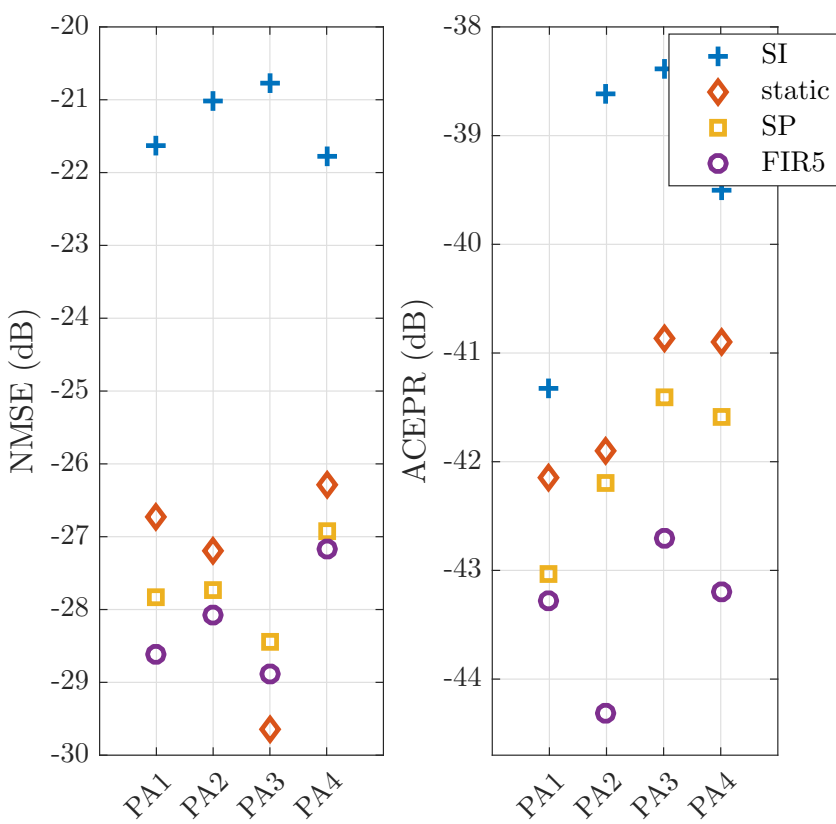

Fig. 10. NMSE (left) and ACEPR (right) of PA output. Results are shown for: the proposed technique with proposed dynamic PA models and singlefrequency S-Parameter antenna array description (SP), the proposed technique with dynamic PA models and filter antenna array description (FIR5), the proposed technique with quasi-static PA models and single-frequency SParameter antenna array description (static), and simulations with single-input PA models (SI).

high-coupling array are given, and in Fig. 11(b) the results for the low-coupling array. In the plots on the left, the spectra of the PA driven in single-path scenario with and without SISO DPD are shown. In this scenario, all out-of-band distortion is due to amplification of $a_{1 i}(n)$. It can be seen that the SISO DPD compensates for the distortion. In the plots on the right, the spectra of the PA driven in MIMO scenario with and without SISO DPD are shown. Two things can be noticed: first, without DPD, the difference between the amount of distortion in single-path scenarios and MIMO scenarios for the different arrays is very small. The distortion due to amplification is higher than the distortion due to crosstalk, such that the crosstalk distortion is masked. Second, by application of SISO DPD, we eliminated the effect of amplitude distortion. Yet, for the MIMO scenario, there is a large amount of out-ofband distortion visible. This means that SISO DPD cannot compensate for the distortion created by crosstalk. As is expected, the distortion due to crosstalk is clearly worse for the high-coupling array. As can be seen in the figure, the simulation results agree with the measurements, which shows that the proposed technique can be used to analyze the effects of crosstalk and mismatch.

\section{CONCLUSIONS}

In this paper we present the derivation of a wideband dual-input PA model which is then utilized in combination with linear dynamic antenna array simulations to predict the characteristics of a multi-antenna TX. The proposed technique 

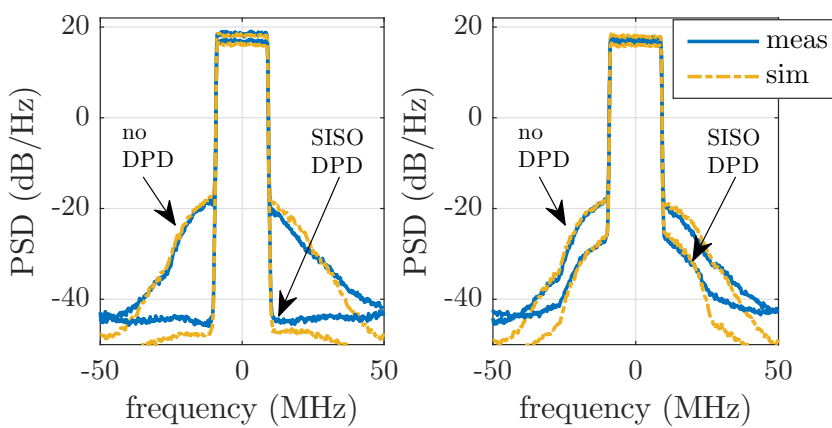

(a)
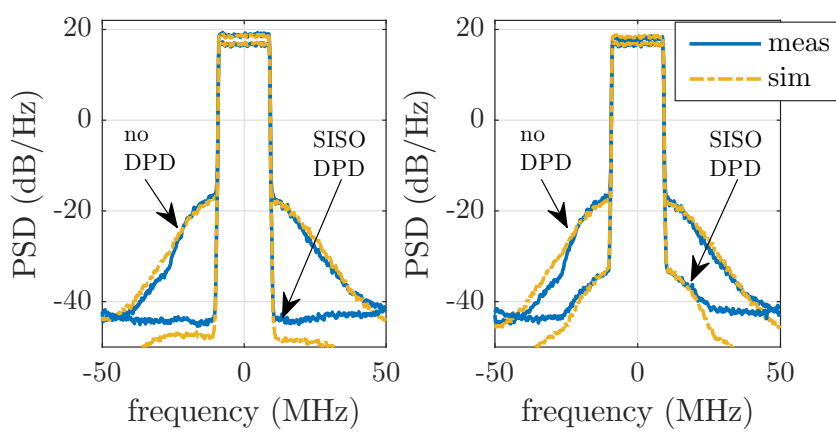

(b)

Fig. 11. Spectra of PA1 for (a) high-coupling array, and (b) low-coupling array. On the left, the PA is operated in a single-path scenario, and on right in a MIMO scenario. Measurements (meas) without SISO DPD and with SISO DPD are compared to simulations (sim) without SISO DPD and with SISO DPD.

allows the output at every antenna of an arbitrarily sized array, as well as the total radiated far-field of the array, to be predicted with only low computational effort. Results are validated in measurements with a four-element TX. The $20 \mathrm{MHz}$ signals used in the validation cause dynamic effects, which define a wideband system, in the PAs as well as the antenna arrays. Hence, our technique can be used as a reliable analysis tool for wideband multi-antenna TXs.

The presented analysis tool can be implemented by designing and characterizing only two main components: The antenna array and the PAs. For our evaluation, we use VNA measurements to determine the characteristics of the antenna arrays. However, the antenna array can be designed in dedicated software to obtain the array scattering matrices and the far-field pattern. An actual fabrication of the antenna array is not necessarily required. For the PA characterization we employ a mixed-mode active load-pull measurement setup to emulate a PA in a multi-antenna TX scenario. With this setup it is possible to acquire the data that is required to identify the dual-input PA model coefficients. Also in this case, the PA data could be obtained from CAD simulations in the transmitter design stage.

With our technique, it is possible to investigate the effects of different antenna arrays on system performance without complicated and costly, sometimes even infeasible, experiments. While the presented demonstrator results are for a multi-antenna TX operated as in wireless communications- based MIMO systems, where each TX path is driven with independent input signals, the presented analysis can equally well be applied for any input signal combination. For example, a very important application of the proposed tool could be for the analysis of highly integrated millimeter wave MIMO and phased array radar TX chips. The complexity, density and interconnect challenges in such applications prevent any in-circuit measurements of the full chip to be performed. The proposed integration of characterization and modeling of sub-circuits, passive interconnects, and antenna elements as described in this work could therefore lay the foundation for the design of such circuits. Hence, applications whose design process could benefit from our work range from high-performance low-cost wireless communication systems employing (massive) MIMO to radar applications.

\section{APPENDIX}

\section{A. Volterra-Series Based Dual Input PA Model for Multi- Antenna TXs}

A single-input low-pass equivalent Volterra model up to nonlinear order $P$ with input $a_{1}(n)$ and output $b_{2}(n)$ is given by [25]

$$
\begin{aligned}
b_{2}(n)= & \sum_{m_{1}=0}^{M} \alpha_{m_{1}}^{(1)} a_{1}\left(n-m_{k}\right)+\sum_{p=2}^{(P-1) / 2+1}[ \\
& \sum_{m_{1}=0}^{M} \ldots \sum_{m_{p}=m_{p-1}}^{M} \sum_{m_{p+1}=0}^{M} \cdots \sum_{m_{2 p-1}=m_{2 p-2}}^{M} \\
& \alpha_{m_{1}, m_{2}, \ldots, m_{2 p-1}}^{(2 p-1)} \\
& \left.\times \prod_{k=1}^{p} a_{1}\left(n-m_{k}\right) \prod_{l=p+1}^{2 p-1} a_{1}^{*}\left(n-m_{l}\right)\right]
\end{aligned}
$$

where $P$ is odd and $M$ is the memory depth. As can be seen, only odd order combinations of the input signal $a_{1}(n)$ need to be considered in the baseband model, where each combination contains exactly one less conjugate term than non-conjugate terms.

We want to obtain a baseband dual-input Volterra seriesbased model suitable for multi-antenna TXs, where only linear terms of the second input occur. Assuming that the two RF input signals are located around the same carrier frequency, (18) can be generalized to the low-pass equivalent of such a dual-input Volterra model with inputs $a_{1}(n)$ and $a_{2}(n)$. This is done by adding all necessary combinations of $a_{1}(n)$ and $a_{2}(n)$ and their conjugates to the model in (18). These are all odd-order combinations where $a_{2}(n)$ occurs only in linear terms, and where the total number of conjugate terms is one less than the total number of non-conjugate terms. The dualinput Volterra model for multi-antenna TX is given by

$$
\begin{aligned}
b_{2}(n)= & \sum_{m_{1}=0}^{M} \alpha_{m_{1}}^{(1)} a_{1}\left(n-m_{k}\right)+\sum_{p=2}^{(P-1) / 2+1}[ \\
& \sum_{m_{1}=0}^{M} \ldots \sum_{m_{p}=m_{p-1}}^{M} \sum_{m_{p+1}=0}^{M} \ldots \sum_{m_{2 p-1}=m_{2 p-2}}^{M} \\
& \alpha_{m_{1}, m_{2}, \ldots, m_{2 p-1}}^{(2 p-1)}
\end{aligned}
$$




$$
\begin{aligned}
& \left.\times \prod_{k=1}^{p} a_{1}\left(n-m_{k}\right) \prod_{l=p+1}^{2 p-1} a_{1}^{*}\left(n-m_{l}\right)\right] \\
& +\sum_{m_{1}=0}^{M} \beta_{m_{1}}^{(1)} a_{2}\left(n-m_{k}\right)+\sum_{p=2}^{(P-1) / 2+1}[ \\
& \sum_{m_{1}=0}^{M} \sum_{m_{2}=0}^{M} \ldots \sum_{m_{p}=m_{p-1}}^{M} \sum_{m_{p+1}=0}^{M} \ldots \sum_{m_{2 p}=m_{2 p-2}}^{M} \\
& \beta_{m_{1}, m_{2}, \ldots, m_{2 p-1}}^{(2 p-1)} a_{2}\left(n-m_{1}\right) \\
& \left.\times \prod_{k=2}^{p} a_{1}\left(n-m_{k}\right) \prod_{l=p+1}^{2 p-1} a_{1}^{*}\left(n-m_{l}\right)\right] \\
& +\sum_{p=2}^{(P-1) / 2+1}\left[\sum_{m_{1}=0}^{M} \sum_{m_{2}=0}^{M} \ldots \sum_{m_{p+1}=m_{p}}^{M}\right. \\
& \sum_{m_{p+2}=0}^{M} \cdots \sum_{m_{2 p}-1=m_{2 p-2}}^{M} \gamma_{m_{1}, m_{2}, \ldots, m_{2 p-1}}^{(2 p-1)} \\
& \left.\times a_{2}^{*}\left(n-m_{1}\right) \prod_{k=2}^{p+1} a_{1}\left(n-m_{k}\right) \prod_{l=p+2}^{2 p-1} a_{1}^{*}\left(n-m_{l}\right)\right]
\end{aligned}
$$

\section{B. Least-Squares Identification of Model Coefficients}

As explained in Section V-B1, the linear least-squares method can be used to estimate the model coefficients $\alpha, \beta$ and $\gamma$ from measurement data, i.e., the measured data vectors $\mathbf{a}_{1}=$ $\left[a_{1}(0), \ldots, a_{1}(N-1)\right]^{T}, \mathbf{a}_{2}=\left[a_{2}(0), \ldots, a_{2}(N-1)\right]^{T}$ and $\mathbf{b}_{\mathbf{2}}=\left[b_{2}(0), \ldots, b_{2}(N-1)\right]^{T}$. The linear least-squares method can be used for all dual-input models given in this work, i.e., the full Volterra series-based model in (3) and (19), the model with memory polynomial structure in (4) and the memoryless model in (5). Using the measured signals, the model output is written in matrix form as

$$
\mathbf{b}_{\mathbf{2}}=\left[\begin{array}{lll}
\mathbf{H}_{\alpha} & \mathbf{H}_{\beta} & \mathbf{H}_{\gamma}
\end{array}\right]\left[\begin{array}{lll}
\boldsymbol{\alpha}^{T} & \boldsymbol{\beta}^{T} & \boldsymbol{\gamma}^{T}
\end{array}\right]^{T} .
$$

Each row of the matrix $\mathbf{H}_{\alpha}$ comprises all terms that contain combinations of $a_{1}(n)$ and $a_{1}^{*}(n)$, e.g. $a_{1}(n), a_{1}(n)\left|a_{1}(n)\right|^{2}$, $a_{1}(n-1), a_{1}(n-1)\left|a_{1}(n-1)\right|^{2}$ and so on, where each column comprises these values for one specific $n$ with $n=$ $0, \ldots, N-1$. In the same manner, the matrix $\mathbf{H}_{\beta}$ contains all combinations which include $a_{2}(n)$, e.g. $a_{2}(n), a_{2}(n-1)$, $a_{2}(n)\left|a_{1}(n)\right|^{2}, a_{2}(n)\left|a_{1}(n-1)\right|^{2}$, etc. In the matrix $\mathbf{H}_{\gamma}$, the terms where $a_{2}^{*}(n)$ occurs, e.g $a_{2}^{*}(n)\left(a_{1}(n)\right)^{2}, a_{2}^{*}(n)\left(a_{1}(n-\right.$ $1))^{2}, a_{2}^{*}(n)\left(a_{1}(n)\right)^{3} a_{1}^{*}(n)$, and so forth, are contained. The vectors $\boldsymbol{\alpha}, \boldsymbol{\beta}$, and $\gamma$ contain the model coefficients, in the sequence that matches the order of the entries in the matrices according to the model structure.

The model coefficients are estimated by transforming (20) using the pseudoinverse with

$$
\left[\begin{array}{lll}
\boldsymbol{\alpha}^{T} & \boldsymbol{\beta}^{T} & \gamma^{T}
\end{array}\right]^{T}=\left[\begin{array}{lll}
\mathbf{H}_{\alpha} & \mathbf{H}_{\beta} & \mathbf{H}_{\gamma}
\end{array}\right]^{+} \mathbf{b}_{\mathbf{2}} .
$$

\section{Derivations for Step-Wise Solution of Multi-Antenna TX Output}

In order to compute the samples of the output vector $\mathbf{b}_{\mathbf{2}}(n)$, first (1) is introduced in (4). Then, all current samples of $\mathbf{b}_{\mathbf{2}}(n)$ are factored out. Introducing (1) is in (4) yields

$$
\left.\begin{array}{l}
b_{2 i}(n)= \\
\sum_{m_{1}=0}^{M_{1}} \sum_{p=0}^{\left(P_{1}-1\right) / 2} \alpha_{m_{1}}^{(2 p+1)} a_{1 i}\left(n-m_{1}\right) \\
\times\left|a_{1 i}\left(n-m_{1}\right)\right|^{2 p} \\
+\sum_{m_{2}=0}^{M_{2}} \beta_{0}^{(1)} \sum_{m_{2}}^{K}\left(\boldsymbol{\lambda}_{i}(k)\right)^{T} \mathbf{b}_{\mathbf{2}}\left(n-k-m_{2}\right) \\
+\sum_{m_{3}=0}^{M_{3}} \sum_{m_{4}=0}^{M_{4}} \sum_{p=1}^{\left(P_{2}-1\right) / 2} \beta_{m_{4} m_{3}}^{(2 p+1)}\left|a_{1 i}\left(n-m_{4}\right)\right|^{2 p} \\
\quad \times \sum_{k=0}^{K}\left(\boldsymbol{\lambda}_{i}(k)\right)^{T} \mathbf{b}_{\mathbf{2}}\left(n-k-m_{3}\right) \\
+\sum_{m_{5}=0}^{M_{5}} \sum_{m_{6}=0}^{M_{6}} \sum_{p=1}^{\left(P_{3}-1\right) / 2} \gamma_{m_{6} m_{5}}^{(2 p+1)} \\
\times\left(a_{1 i}\left(n-m_{6}\right)\right)^{p+1}\left(a_{1 i}^{*}\left(n-m_{6}\right)\right)^{p-1} \\
\times \sum_{k=0}^{K}\left(\boldsymbol{\lambda}_{i}^{*}(k)\right)^{T} \mathbf{b}_{\mathbf{2}}^{*}\left(n-k-m_{5}\right) .
\end{array}\right\}
$$

In (22a) $\mathbf{b}_{\mathbf{2}}(n)$ does not occur. Hence, first (22b) is transformed into

$$
\begin{aligned}
& \beta_{00}^{(1)}\left(\boldsymbol{\lambda}_{i}(0)\right)^{T} \mathbf{b}_{\mathbf{2}}(n)+\beta_{00}^{(1)} \sum_{k=1}^{K}\left(\boldsymbol{\lambda}_{i}(k)\right)^{T} \mathbf{b}_{\mathbf{2}}(n-k) \\
+ & \sum_{m_{2}=1}^{M_{2}} \beta_{0 m_{2}}^{(1)} \sum_{k=0}^{K}\left(\boldsymbol{\lambda}_{i}(k)\right)^{T} \mathbf{b}_{\mathbf{2}}\left(n-k-m_{2}\right) \\
+ & \left(\boldsymbol{\lambda}_{i}(0)\right)^{T} \mathbf{b}_{\mathbf{2}}(n) \sum_{m_{4}=0}^{M_{4}} \sum_{p=1}^{\left(P_{2}-1\right) / 2} \beta_{m_{4} 0}^{(2 p+1)}\left|a_{1 i}\left(n-m_{4}\right)\right|^{2 p} \\
+ & \sum_{m_{4}=0}^{M_{4}} \sum_{p=1}^{\left(P_{2}-1\right) / 2} \beta_{m_{4} 0}^{(2 p+1)}\left|a_{1 i}\left(n-m_{4}\right)\right|^{2 p} \\
& \times \sum_{k=1}^{K}\left(\boldsymbol{\lambda}_{i}(k)\right)^{T} \mathbf{b}_{\mathbf{2}}(n-k) \\
+ & \sum_{m_{3}=1}^{M_{3}} \sum_{m_{4}=0}^{M_{4}} \sum_{p=1}^{\left(P_{2}-1\right) / 2} \beta_{m_{4} m_{3}}^{(2 p+1)}\left|a_{1 i}\left(n-m_{4}\right)\right|^{2 p} \\
& \times \sum_{k=0}^{K}\left(\boldsymbol{\lambda}_{i}(k)\right)^{T} \mathbf{b}_{\mathbf{2}}\left(n-k-m_{3}\right) .
\end{aligned}
$$

Then, $(22 \mathrm{c})$ is transformed into

$$
\begin{aligned}
& \left(\boldsymbol{\lambda}_{i}^{*}(0)\right)^{T} \mathbf{b}_{\mathbf{2}}^{*}(n) \\
\times & \sum_{m_{6}=0}^{M_{6}} \sum_{p=1}^{\left(P_{3}-1\right) / 2} \gamma_{m_{6} 0}^{(2 p+1)}\left(a_{1 i}\left(n-m_{6}\right)\right)^{p+1}\left(a_{1 i}^{*}\left(n-m_{6}\right)\right)^{p-1} \\
+ & \sum_{m_{6}=0}^{M_{6}} \sum_{p=1}^{\left(P_{3}-1\right) / 2} \gamma_{m_{6} 0}^{(2 p+1)}\left(a_{1 i}\left(n-m_{6}\right)\right)^{p+1}\left(a_{1 i}^{*}\left(n-m_{6}\right)\right)^{p-1} \\
\times & \sum_{k=1}^{K}\left(\boldsymbol{\lambda}_{i}^{*}(k)\right)^{T} \mathbf{b}_{\mathbf{2}}^{*}(n-k)
\end{aligned}
$$




$$
\begin{aligned}
& +\sum_{m_{5}=1}^{M_{5}} \sum_{m_{6}=0}^{M_{6}} \sum_{p=1}^{\left(P_{3}-1\right) / 2} \gamma_{m_{6} m_{5}}^{(2 p+1)} \\
& \times\left(a_{1 i}\left(n-m_{6}\right)\right)^{p+1}\left(a_{1 i}^{*}\left(n-m_{6}\right)\right)^{p-1} \\
& \times \sum_{k=0}^{K}\left(\boldsymbol{\lambda}_{i}^{*}(k)\right)^{T} \mathbf{b}_{\mathbf{2}}^{*}\left(n-k-m_{5}\right) .
\end{aligned}
$$

\section{ACKNOWLEDGMENT}

The authors would like to thank Skyworks Solutions, Inc. for donating the PA test boards used in the experiments.

\section{REFERENCES}

[1] H. Inanoglu, "Multiple-input multiple-output system capacity: Antenna and propagation aspects," IEEE Antennas Propag. Mag., vol. 55, no. 1, pp. 253-273, 2013.

[2] E. Larsson, O. Edfors, F. Tufvesson, and T. Marzetta, "Massive MIMO for next generation wireless systems," IEEE Commun. Mag., vol. 52, no. 2, pp. 186-195, February 2014.

[3] F. Rusek, D. Persson, B. K. Lau, E. Larsson, T. Marzetta, O. Edfors, and F. Tufvesson, "Scaling up MIMO: Opportunities and challenges with very large arrays," IEEE Signal Process. Mag., vol. 30, no. 1, pp. 40-60, Jan 2013.

[4] M. Romier, A. Barka, H. Aubert, J.-P. Martinaud, and M. Soiron, "Load-pull effect on radiation characteristics of active antennas," IEEE Antennas Wireless Propag. Lett., vol. 7, pp. 550-552, 2008.

[5] S. Bassam, M. Helaoui, and F. Ghannouchi, "Crossover digital predistorter for the compensation of crosstalk and nonlinearity in MIMO transmitters," IEEE Trans. Microw. Theory Tech., vol. 57, no. 5, pp. 1119-1128, May 2009.

[6] S. Amin, P. Landin, P. Handel, and D. Ronnow, "Behavioral modeling and linearization of crosstalk and memory effects in RF MIMO transmitters," IEEE Trans. Microw. Theory Tech., vol. 62, no. 4, pp. 810-823, April 2014.

[7] S. Farsi, J. Dooley, K. Finnerty, D. Schreurs, B. Nauwelaers, and R. Farrell, "Behavioral modeling approach for array of amplifiers in active antenna array system," in IEEE Topical Conf. on Power Amplifiers for Wireless and Radio Applications, Jan 2013, pp. 73-75.

[8] J. Qi and S. Aissa, "Analysis and compensation for the joint effects of hpa nonlinearity, I/Q imbalance and crosstalk in MIMO beamforming systems," in IEEE Wireless Commun. Networking Conf., 2011, pp. 15621567.

[9] J. Kim and K. Konstantinou, "Digital predistortion of wideband signals based on power amplifier model with memory," Electron. Lett., vol. 37, no. 23, pp. 1417-1418, Nov 2001

[10] G. El Nashef, F. Torres, S. Mons, T. Reveyrand, T. Monediere, E. Ngoya, and R. Quere, "EM/circuit mixed simulation technique for an active antenna," IEEE Antennas Wireless Propag. Lett., vol. 10, pp. 354-357, May 2011.

[11] D. Root, J. Verspecht, D. Sharrit, J. Wood, and A. Cognata, "Broad-band poly-harmonic distortion (PHD) behavioral models from fast automated simulations and large-signal vectorial network measurements," IEEE Trans. Microw. Theory Tech., vol. 53, no. 11, pp. 3656-3664, 2005.

[12] H. Zargar, A. Banai, and J. Pedro, "A new double input-double output complex envelope amplifier behavioral model taking into account source and load mismatch effects," IEEE Trans. Microw. Theory Tech., vol. 63, no. 2, pp. 766-774, Feb 2015.

[13] C. Fager, X. Bland, K. Hausmair, J. Cahuana, and T. Eriksson, "Prediction of smart antenna transmitter characteristics using a new behavioral modeling approach," in IEEE MTT-S Int. Microw. Symp.(IMS), June 2014, pp. 1-4.

[14] J. Pedro and S. Maas, "A comparative overview of microwave and wireless power-amplifier behavioral modeling approaches," IEEE Trans. Microw. Theory Tech., vol. 53, no. 4, pp. 1150-1163, 2005.

[15] M. Schetzen, The Volterra and Wiener theories of nonlinear systems, 2nd ed. Krieger Publishing Company, Malabar, Florida, 2006.

[16] G. Z. El Nashef, F. Torres, S. Mons, T. Reveyrand, T. Monediere, E. N'Goya, and R. Quere, "Second order extension of power amplifiers behavioral models for accuracy improvements," in European Microw. Conf., Sept 2010, pp. 1030-1033.
[17] D. Morgan, Z. Ma, J. Kim, M. Zierdt, and J. Pastalan, "A generalized memory polynomial model for digital predistortion of RF power amplifiers," IEEE Trans. Signal Process., vol. 54, no. 10, pp. 3852-3860, Oct 2006.

[18] A. Zhu, J. Pedro, and T. Brazil, "Dynamic deviation reduction-based volterra behavioral modeling of RF power amplifiers," IEEE Trans. Microw. Theory Tech., vol. 54, no. 12, pp. 4323-4332, Dec 2006.

[19] C. Fager, K. Hausmair, K.and Andersson, E. Sienkiewicz, D. Gustafsson, and K. Buisman, "Analysis of nonlinear distortion in phased array transmitters," in Int. Workshop on Integr. Nonlinear Microw. and Millimetrewave Circ., April 2017, accepted for publication.

[20] H. Zargar, A. Banai, and J. C. Pedro, "DIDO behavioral model extraction setup using uncorrelated envelope signals," in European Microw. Conf., Sept 2015, pp. 646-649.

[21] S. Gustafsson, M. Thorsell, and C. Fager, "A novel active load-pull system with multi-band capabilities," in ARFTG Microw. Meas. Conf., 2013.

[22] K. Andersson and C. Fager, "Oscilloscope based two-port measurement system using error-corrected modulated signals," in Workshop Integr. Nonlinear Microw. Millimetre-Wave Circuits, Sept 2012, pp. 1-3.

[23] M. Isaksson, D. Wisell, and D. Ronnow, "A comparative analysis of behavioral models for RF power amplifiers," IEEE Trans. Microw. Theory Tech., vol. 54, no. 1, pp. 348-359, Jan 2006.

[24] S. Afsardoost, T. Eriksson, and C. Fager, "Digital predistortion using a vector-switched model," IEEE Trans. Microw. Theory Tech., vol. 60, no. 4, pp. 1166-1174, 2012.

[25] E. Lima, T. Cunha, H. Teixeira, M. Pirola, and J. Pedro, "Base-band derived volterra series for power amplifier modeling," in IEEE MTT-S Int. Microw. Symp. Dig., June 2009, pp. 1361-1364.

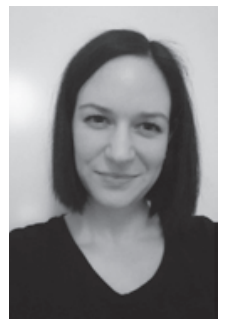

Katharina Hausmair received the Dipl.-Ing. degree in electrical and information engineering from Graz University of Technology, Austria, in 2010. From 2010-2013 she was a researcher at the Signal Processing and Speech Communication Laboratory of Graz University of Technology. Since 2013, she has been with the Department of Signals and Systems at Chalmers University of Technology, Göteborg, Sweden, pursuing a Ph.D. degree. Her research interests include signal processing for communication systems with emphasis on modeling and compensation of undesired effects occurring in analog circuits.

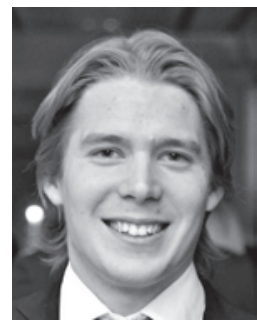

Sebastian Gustafsson (S'12) received the M.Sc. degree in electrical engineering from Chalmers University of Technology, Göteborg, Sweden, in 2013. In 2013 he joined the Microwave Electronics Laboratory group at Chalmers University of Technology as a Ph.D. student. His interests include RF metrology and GaN HEMT characterization.

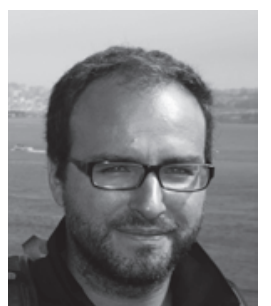

César Sánchez-Pérez received the $\mathrm{Ph} . \mathrm{D}$. degree from the University of Zaragoza, Spain in 2012. From September 2012 to December 2014, he worked as a postdoc at the Microwave Electronics Laboratory, Chalmers University of Technology, Sweden. Since January 2015 he works as RF/microwave specialist at Qamcom Research Technology AB in Sweden. His research interests include wireless communications systems, with emphasis on tunable matching networks and high efficiency transmitters. 


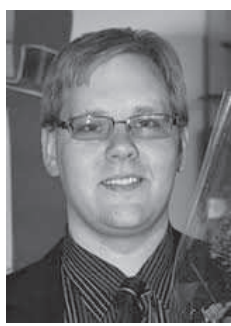

Per N. Landin received the M.Sc. degree from Uppsala University, Uppsala, Sweden, in 2007, and the Ph.D. degree jointly from the KTH Royal Institute of Technology, Stockholm, Sweden, and Vrije Universiteit Brussel, Brussels, Belgium, in 2012. During 2013-2014 he was a post-doctoral researcher at Chalmers University of Technology, Göteborg, Sweden. Since 2015 he is with Ericsson AB, Kumla, Sweden. His main research interests are RF measurements and signal processing, over-the-air meaamplifier modeling and linearization.

surements and system identification applied to power

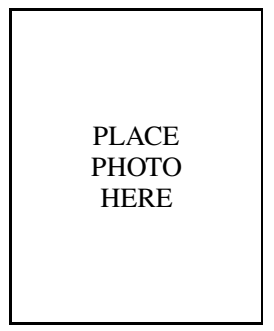

Ulf Gustavsson received a M.Sc. degree in electrical engineering from Örebro University, Sweden, in 2006, and a Ph.D. degree from Chalmers University of Technology, Sweden, in 2011. He is currently a Senior Researcher with Ericsson AB Research, Göteborg. He is also the Lead Scientist for Ericsson AB Research within the Marie Skłodowska-Curie Innovative Training Network, SILIKA. His main research interests lie in radio signal processing and behavioral modeling of radio hardware for advanced antenna systems.

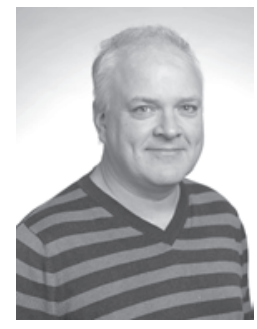

Thomas Eriksson received the Ph.D. degree in Information Theory from Chalmers University of Technology, Göteborg, Sweden, in 1996. From 1990 to 1996, he was at Chalmers. In 1997 and 1998, he was at AT\&T Labs - Research, Murray Hill, NJ, USA. In 1998 and 1999, he was at Ericsson Radio Systems AB, Kista, Sweden. Since 1999, he has been with Chalmers University, where he is currently a professor of communication systems. Further, he was a guest professor with Yonsei University, S. Korea, in 2003-2004. He has authored or co-authored more than 200 journal and conference papers, and holds 11 patents. Prof. Eriksson is leading the research on hardware-constrained communications with Chalmers University of Technology. His research interests include communication, data compression, and modeling and compensation of non-ideal hardware components (e.g. amplifiers, oscillators, and modulators in communication transmitters and receivers, including massive MIMO). Currently, he is leading several projects on e.g. 1) massive MIMO communications with imperfect hardware, 2) MIMO communication taken to its limits: 100Gbit/s link demonstration, 3) Massive MIMO testbed design, 4), Satellite communication with phase noise limitations, 5) Efficient and linear transceivers, etc. He is currently the Vice Head of the Department of Signals and Systems with Chalmers University of Technology, where he is responsible for undergraduate and Master's education.

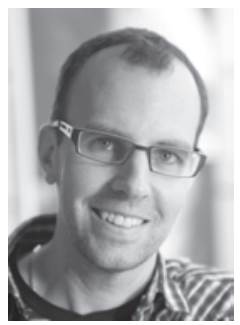

Christian Fager received his Ph.D. degree from Chalmers University of Technology, Sweden, in 2003. Since 2015, he is a Professor at the Microwave Electronics Laboratory at the same university where he is also Deputy Director of the $\mathrm{GHz}$ Centre for industrial collaborations.

Dr. Fager has authored and co-authored more than 120 publications in international journals and conferences. His research is focused in the area of energy efficient and linear transmitters for future wireless communication systems. Dr. Fager received the Best Student Paper Award at the IEEE International Microwave Symposium in 2003. He serves as a TPC member of the IEEE IMS and INMMiC technical conferences and is currently an Associate Editor of IEEE Microwave Magazine. 